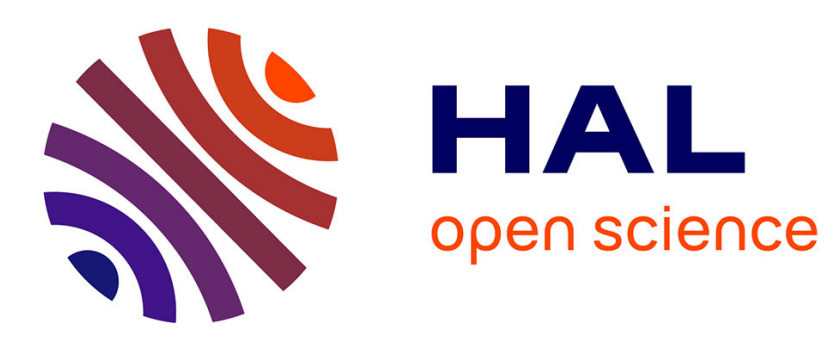

\title{
Minimum time control of the restricted three-body problem
}

\author{
Jean-Baptiste Caillau, Bilel Daoud
}

\section{To cite this version:}

Jean-Baptiste Caillau, Bilel Daoud. Minimum time control of the restricted three-body problem. SIAM Journal on Control and Optimization, 2012, 50 (6), pp.3178-3202. hal-00599216v4

\section{HAL Id: hal-00599216 https://hal.science/hal-00599216v4}

Submitted on 10 Apr 2012

HAL is a multi-disciplinary open access archive for the deposit and dissemination of scientific research documents, whether they are published or not. The documents may come from teaching and research institutions in France or abroad, or from public or private research centers.
L'archive ouverte pluridisciplinaire HAL, est destinée au dépôt et à la diffusion de documents scientifiques de niveau recherche, publiés ou non, émanant des établissements d'enseignement et de recherche français ou étrangers, des laboratoires publics ou privés. 


\title{
Minimum time control of the restricted three-body problem
}

\author{
J.-B. Caillau* $\quad$ B. Daoud ${ }^{\dagger}$
}

July 2011

\begin{abstract}
The minimum time control of the circular restricted three-body problem is considered. Controllability is proved on an adequate submanifold. Singularities of the extremal flow are studied by means of a stratification of the switching surface. Properties of homotopy maps in optimal control are framed in a simple case. The analysis is used to perform continuations on the two parameters of the problem: The ratio of masses, and the magnitude of the control.
\end{abstract}

Keywords. three-body problem, minimum time control, control-affine systems, homotopy, conjugate points

MSC classification. 49K15, 70Q05

\section{Problem statement}

The control of the two-body problem was addressed in [15. The first body exerted a central force on the second, which was an artificial satellite of negligible mass whose thrust was the control. The resulting controlled Kepler equation was shown to be controllable, and minimization of time was studied (see also the subsequent papers [8, 14]). The present paper is the continuation of this work. Now under the influence of two primary bodies, the artificial satellite is still endowed with a thrust. The motion of the two primaries, not influenced by the third negligible mass, is supposed to be circular. Among the numerous previous studies on space missions in the three-body framework, one has to mention the pioneering work of [19], and more recently of [25]. These approaches are purely celestial mechanical ones and rely on a fine knowledge of the dynamical system with three bodies or more. More on the control side see, e.g., 4 for numerical results using direct methods, [6 for a preliminary study on stabilization, and

\footnotetext{
*Math. Institute, Bourgogne Univ. \& CNRS, 9 avenue Savary, F-21078. Supported by Conseil Régional de Bourgogne (contract no. 2009-160E-160-CE-160T), ANR Geometric Control Methods (project no. NT09_504490), and SADCO Initial Training Network (FP7 grant no. 264735-SADCO).

†Same address. Supported by the French Ministry for Higher Education \& Research (grant no. 31716-2008)
} 
27. 28 for a combination of control and dynamical system techniques. We present a purely optimal control approach for time minimization. The indirect methods (shooting) used for numerical computations are driven by the geometric analysis of the problem. The model we consider is the following 33 .

Let $\mu \in(0,1)$ be the ratio of the primaries masses, and let $Q_{\mu}:=\mathbf{C} \backslash\{-\mu, 1-$ $\mu\}$. For $q \in Q_{\mu} \subset \mathbf{C} \simeq \mathbf{R}^{2}$ and positive thrust magnitude $\varepsilon$, define the controlled circular restricted three-body problem (planar model) according to

$$
\ddot{q}(t)-\nabla \Omega_{\mu}(q(t))+2 i \dot{q}(t)=\varepsilon u(t), \quad|u(t)|=\sqrt{u_{1}^{2}(t)+u_{2}^{2}(t)} \leq 1 .
$$

Here, $(q, \dot{q}) \in X_{\mu}=T Q_{\mu} \simeq Q_{\mu} \times \mathbf{C}$ are Cartesian coordinates in a rotating frame $\left(q=e^{-i t} \xi\right.$ where $\xi$ is the position vector in a fixed frame) and

$$
\Omega_{\mu}(q):=\frac{1}{2}|q|^{2}+\frac{1-\mu}{|q+\mu|}+\frac{\mu}{|q-1+\mu|} .
$$

Another choice of coordinates consists in letting $X_{\mu}=T^{*} Q_{\mu}$, taking the cotangent bundle instead to write the uncontrolled part of the dynamics in Hamiltonian form. Let $p=\dot{q}+i q$ and let

$$
\begin{aligned}
J_{\mu}(q, \dot{q}) & :=\frac{1}{2}|\dot{q}|^{2}-\Omega_{\mu}(q), \\
& =\frac{1}{2}|p|^{2}+p \wedge q-\frac{1-\mu}{|q+\mu|}-\frac{\mu}{|q-1+\mu|}
\end{aligned}
$$

be the Jacobian integral. Then,

$$
\dot{q}(t)=\frac{\partial J_{\mu}}{\partial p}(q(t), p(t)), \quad \dot{p}(t)=-\frac{\partial J_{\mu}}{\partial q}(q(t), p(t))+\varepsilon u(t), \quad|u(t)| \leq 1 .
$$

More compactly,

$$
\dot{x}(t)=F_{0}(x(t))+\varepsilon u_{1}(t) F_{1}(x(t))+\varepsilon u_{2}(t) F_{2}(x(t)), \quad|u(t)| \leq 1,
$$

with, in $(q, p)$ coordinates for $x \in X_{\mu}$,

$$
F_{0}(q, p):=\overrightarrow{J_{\mu}}, \quad F_{1}(q, p):=\frac{\partial}{\partial p_{1}}, \quad F_{2}(q, p):=\frac{\partial}{\partial p_{2}}
$$

where the symplectic gradient $\overrightarrow{J_{\mu}}=\left(\nabla_{p} J_{\mu},-\nabla_{q} J_{\mu}\right)$ is the drift of the system. When $\mu=0$, we get a two-body problem: $J_{0}=E-C$ with energy and momentum respectively equal to

$$
\begin{gathered}
E:=\frac{1}{2}|\dot{\xi}|^{2}-\frac{1}{|\xi|}=\frac{1}{2}|\dot{q}|^{2}-\frac{1}{2}|q|^{2}-q \wedge \dot{q}-\frac{1}{|q|} \\
C:=\xi \wedge \dot{\xi}=q \wedge \dot{q}+|q|^{2} .
\end{gathered}
$$

Restricting to the elliptic domain, $X_{0} \cap\{E<0, C>0\}$, another system of coordinates tailored for the analysis is obtained using orbital elements describing the geometry of the osculating ellipse. Let $n>0$ be the mean motion $\left(a^{3} n^{2}=1\right.$ if $a$ the semi-major axis), $\left(e_{x}, e_{y}\right) \in \mathbf{D}$ be the eccentricity vector (where $\mathbf{D}$ is the open unit ball of $\mathbf{R}^{2}$ ), and $l \in \mathbf{R}$ the longitude (the class modulo $2 \pi$ of $l$ is 
just the polar angle in the fixed $\left(\xi_{1}, \xi_{2}\right)$-frame). Alternatively, one can use polar coordinates $(e, \theta) \in(0,1) \times \mathbf{S}^{1}$ for the eccentricity on the (pointed) Poincaré disk $\mathbf{D}(\theta$ is called the argument of pericenter $)$. In this system, $x=(n, e, \theta, l)$,

$$
\begin{gathered}
F_{0}(x)_{\mid \mu=0}=\frac{n W^{2}}{\left(1-e^{2}\right)^{3 / 2}} \frac{\partial}{\partial l}, \quad W=1+e \cos \tau \\
\widetilde{F}_{1}(x)=\frac{\sqrt{1-e^{2}}}{n^{1 / 3}}\left(-\frac{3 n e \sin \theta}{1-e^{2}} \frac{\partial}{\partial n}+\sin \tau \frac{\partial}{\partial e}-\cos \tau \frac{1}{e} \frac{\partial}{\partial \theta}\right), \\
\widetilde{F}_{2}(x)=\frac{\sqrt{1-e^{2}}}{n^{1 / 3}}\left(-\frac{3 n W}{1-e^{2}} \frac{\partial}{\partial n}+\left(\cos \tau+\frac{e+\cos \tau}{W}\right) \frac{\partial}{\partial e}+\left(\sin \tau+\frac{\sin \tau}{W}\right) \frac{1}{e} \frac{\partial}{\partial \theta}\right)
\end{gathered}
$$

where $\tau=l-\theta$. We have also used a feedback on the control to express the control not in the $\left\{\partial / \partial \dot{\xi}_{1}, \partial / \partial \dot{\xi}_{2}\right\}$ frame but in the radial-orthoradial one, so

$$
\widetilde{F}_{1}(\xi, \dot{\xi})=\frac{\xi_{1}}{|\xi|} \frac{\partial}{\partial \dot{\xi}_{1}}+\frac{\xi_{2}}{|\xi|} \frac{\partial}{\partial \dot{\xi}_{2}}, \quad \widetilde{F}_{2}(\xi, \dot{\xi})=-\frac{\xi_{2}}{|\xi|} \frac{\partial}{\partial \dot{\xi}_{1}}+\frac{\xi_{1}}{|\xi|} \frac{\partial}{\partial \dot{\xi}_{2}} .
$$

The criterion under consideration is the final time, and the paper is organized as follows.

Section 2 is devoted to controllability. Independently of the bound on the control, it is proved that admissible trajectories between arbitrary points exist provided the Jacobian integral is smaller than the one given for some equilibrium point of the uncontrolled system. The structure of optimal controls is addressed in Section 3, refining the results of [15. In particular the role of peri and apocenters with respect to global bounds on the number of switchings of the control is emphasized, in connection with averaging of the system. The system has indeed two parameters: The bound of the control, $\varepsilon$, which can be taken very small with low-thrust applications in mind 29] thus leading to averaging; and the ratio of masses, $\mu$, on which a continuation à la Poincaré may be performed to embed the two-body problem into a three-body one. This idea is the key to solve the problem as explained in Section 4 where continuations both with respect to $\mu$ and $\varepsilon$ are considered. The peculiarity of homotopy maps in optimal control is then illustrated in a simple framework, in relation to second order optimality conditions.

\section{Controllability}

The drift has five equilibrium points, $L_{1}(\mu), \ldots, L_{5}(\mu)$, known as Lagrange points [33, whose position depends on $\mu$. The points $L_{1}, L_{2}$ and $L_{3}$ are the collinear or Euler points, the points $L_{4}$ and $L_{5}$ form two equilateral triangles with $-\mu$ and $1-\mu$. When $\mu=0, L_{1}=L_{2}=1, L_{3}=-1$, and $L_{4}=\exp (i \pi / 3), L_{5}=-\exp (i \pi / 3)$ (all belong to $\mathbf{S}^{1}$ which is a continuum of equilibrium points in this particular case). The Jacobian $J_{\mu}$ is the only first integral of the non-integrable uncontrolled system. Every level set $\left\{J_{\mu}=j\right\}$ projects onto $\Omega_{\mu}(q)+j=|\dot{q}|^{2} / 2 \geq 0$ in the $\left(q_{1}, q_{2}\right)$-space, defining the Hill regions where the free motion has to take place (see Fig. 1). Let $j_{i}(\mu):=J_{\mu}\left(L_{i}(\mu)\right.$ ), $i=1, \ldots, 5$ denote the Jacobian constants of these points. For $\mu \in(0,1)$, $j_{2}<j_{1}<j_{3}<j_{4}=j_{5}$ (these values all degenerate to $-3 / 2$ when $\mu$ goes to 


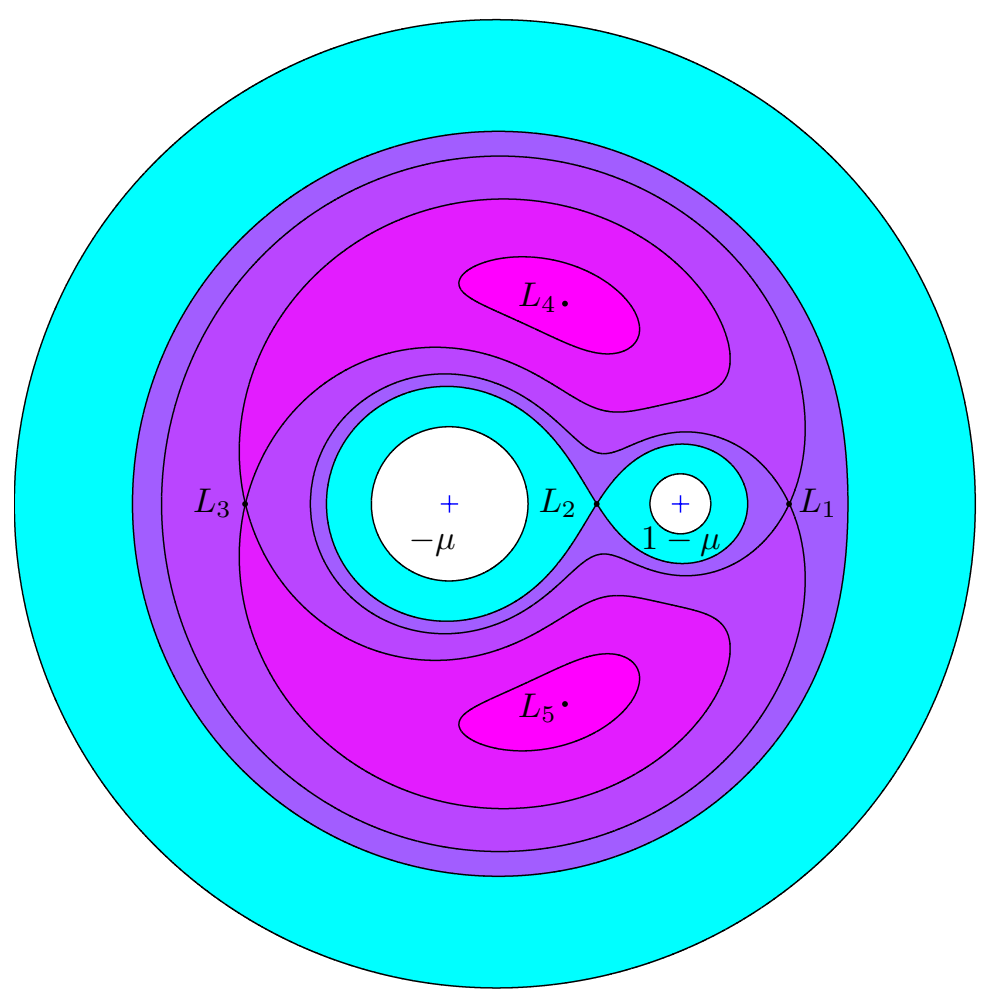

Figure 1: Lagrange points and associated Hill regions, $\mu \in(0,1)$. The forbidden regions of motion (complementary to the Hill regions) monotonically decrease as the Jacobian constant tends to $J_{\mu}\left(L_{4}\right)=J_{\mu}\left(L_{5}\right)$ (then disappear past this value) and opens up past $J_{\mu}\left(L_{1}\right)$.

zero). The open subset $\left\{x \in X_{\mu} \mid J_{\mu}(x)<j_{1}(\mu)\right\}$ has two connected components, and we denote by $X_{\mu}^{1}$ the component containing $L_{2}(\mu)$ (see Fig. 2). The result below essentially asserts that controllability for the restricted three-body problem holds provided the Jacobian is less than the Jacobian at $L_{1}$, emphasizing the role of Lagrange points. (Regarding the role of $L_{2}$, see Fig. 5 and the numerical results in Section 4, )

Theorem 1. For any $\mu \in(0,1)$, for any positive $\varepsilon$, the circular restricted three-body problem is controllable on $X_{\mu}^{1}$.

We postpone the proof to the end of the section and first recall some basic facts needed to assert controllability.

Consider a smooth ${ }^{1}$ control-affine system on a manifold $X$,

$$
\dot{x}(t)=F_{0}(x(t))+\sum_{i=1}^{m} u_{i} F_{i}(x(t)), \quad u(t) \in U \subset \mathbf{R}^{m},
$$

such that $U$ is a neighbourhood of the origin. The attainable set (by piecewise constant controls) from $x_{0} \in X$ depends only on the drift, $F_{0}$, and on the

\footnotetext{
${ }^{1}$ That is $\mathscr{C}^{\infty}$-smooth.
} 
distribution $\mathscr{D}$ spanned by the vector fields $F_{1}, \ldots, F_{m}$. It is the set of points obtained by compositions of flows,

$$
e^{t_{p} G_{p}} \circ \cdots \circ e^{t_{1} G_{1}}\left(x_{0}\right), \quad G_{i} \in F_{0}+\mathscr{D}, \quad t_{i} \geq 0
$$

with $t_{i}$ small enough for the composition to be defined. Now, if $\mathscr{F}$ is an arbitrary subsheaf of $\mathscr{C}^{\infty}$ vector fields on $X$, assuming for simplicity all $F \in \mathscr{F}$ complete, define the subgroup $\mathscr{G}$ of the diffeomorphisms of $X$ generated by the one parameter subgroups $\exp t F, t \in \mathbf{R}, F \in \mathscr{F}$. According to the orbit theorem [1, 31, the orbit of $\mathscr{G}$ through $x_{0}$ is an immersed submanifold of $X$ whose tangent space is

$$
T_{x} \mathscr{G}\left(x_{0}\right)=\operatorname{Span}_{x}\{(\operatorname{Ad} \varphi) F, \varphi \in \mathscr{G}, F \in \mathscr{F}\} .
$$

In coordinates,

$$
(\operatorname{Ad} \varphi) F_{\mid x}=\left[\varphi^{\prime}(x)\right]^{-1} F(\varphi(x)), \quad x \in X .
$$

Restricting to the $\mathscr{C}^{\omega}$-category, the adjoint action and the exponential commute in the sense that, for arbitrary vector fields $F, G$,

$$
\left(\operatorname{Ad} e^{t F}\right) G_{\mid x}=\left(e^{t \operatorname{ad} F}\right) G_{\mid x}=\sum_{n \geq 0} \frac{t^{n}}{n !}\left(\operatorname{ad}^{n} F\right) G_{\mid x}, \quad x \in X
$$

where $(\operatorname{ad} F) G$ is the Lie bracket $[F, G]=F \cdot G-G \cdot F$, and recursively for $\operatorname{ad}^{n}$. In this case, the orbit theorem then simply reads

$$
T_{x} \mathscr{G}\left(x_{0}\right)=\operatorname{Lie}_{x} \mathscr{F} .
$$

If the vector fields are not complete, $\mathscr{G}$ is just a pseudo-group 23 but the conclusion of the orbit theorem - which is local - is preserved. Coming back to control-affine systems, assuming that

$$
\operatorname{Lie}_{x}\left\{F_{0}, F_{1}, \ldots, F_{m}\right\}=T_{x} X, \quad x \in X,
$$

and that the drift $F_{0}$ is recurrent ${ }^{2}$ it can be proven that compositions with the flow of $-F_{0}$ can be added when computing the attainable set (see [21]). So the attainable set is equal to the orbit of the pseudo-group associated with $\left\{F_{0}, F_{1}, \ldots, F_{m}\right\}$. Because of the orbit theorem and of $(1)$, this orbit has to be the whole manifold (supposed to be connected). In the control-affine three-body case, the rank condition holds as is clear from

Lemma 1. A second order controlled system on $\mathbf{R}^{m}$,

$$
\ddot{q}(t)+g(q(t), \dot{q}(t))=u(t),
$$

is a control-affine system on $\mathbf{R}^{2 m}$ with an involutive distribution $\mathscr{D}$ and a drift $F_{0}$ such that $\left\{F_{1}, \ldots, F_{m},\left[F_{0}, F_{1}\right], \ldots,\left[F_{0}, F_{m}\right]\right\}$ has maximum rank.

\footnotetext{
${ }^{2}$ Given a vector field $F$, a point $x \in X$ is recurrent or positively Poisson stable for $F$ if, for any neighbourhood $V$ of $x$, for any positive $T$, there is $t \geq T$ such that $\exp t F(x)$ is defined and belongs to $V$. The vector field itself is said recurrent when it has a dense subset of recurrent points.
} 
Proof. As a first order system,

$$
F_{0}(q, \dot{q})=\dot{q}_{1} \frac{\partial}{\partial q_{1}}+\cdots+\dot{q}_{m} \frac{\partial}{\partial q_{m}}-g_{1}(q, \dot{q}) \frac{\partial}{\partial \dot{q}_{1}}-\cdots-g_{m}(q, \dot{q}) \frac{\partial}{\partial \dot{q}_{m}}
$$

and $F_{i}=\partial / \partial \dot{q}_{i}$ (so $\mathscr{D}$ is clearly involutive). Then

$$
\left[F_{0}, F_{i}\right]=-\partial / \partial q_{i} \quad \bmod \mathscr{D}
$$

and the rank is maximum.

Applying the lemma to the planar three-body problem $(m=2)$, one obtains controllability in the following way.

Proof (of Theorem 1). Let $x_{0}, x_{f}$ in $X_{\mu}^{1}$, and let $j$ be strictly smaller than the Jacobian constants of both endpoints. Set $\widetilde{X}_{\mu}^{1}:=X_{\mu}^{1} \cap\left\{J_{\mu}>j\right\}$. Outside a subset of zero measure associated with initial conditions generating collisions $(q=-\mu$ or $1-\mu)$, the drift is a complete Hamiltonian vector field whose exponential is defined for all times and is a volume preserving bijection in $(q, p)$ coordinates. By definition, $\widetilde{X}_{\mu}^{1}$ which is a union of level sets of the Hamiltonian $J_{\mu}$ is invariant with respect to the exponential. For $x=(q, p) \in \widetilde{X}_{\mu}^{1}$,

$$
j+\Omega_{\mu}(q)<\frac{1}{2}|p-i q|^{2}<j_{1}(\mu)+\Omega_{\mu}(q) .
$$

Then, for a fixed $q$, the volume of the $q$-section of $\widetilde{X}_{\mu}^{1}$ is bounded by $2 \pi\left(j_{1}(\mu)-j\right)$ as is clear integrating with respect to $\mathrm{d} p_{1} \wedge \mathrm{d} p_{2}=\rho \mathrm{d} \rho \wedge \mathrm{d} \alpha$ (set $p-i q=$ : $\rho \exp (i \alpha))$. Since the projection on the $\left(q_{1}, q_{2}\right)$-space of $\widetilde{X}_{\mu}^{1}$ is also bounded, the $\mathrm{d} q \wedge \mathrm{d} p$-measure of $\widetilde{X}_{\mu}^{1}$ is finite (Fubini). We conclude as in 30] that almost every point of $\widetilde{X}_{\mu}^{1}$ is recurrent by Poincaré theorem. Controllability on $\widetilde{X}_{\mu}^{1}$ follows and implies the existence of a trajectory joining $x_{0}$ to $x_{f}$, which in turn implies controllability on $X_{\mu}^{1}$.

Remark 1. In the two-body case, $\mu=0$, controllability still holds on $X_{0}^{1}$, the bounded component of $\left\{J_{0}<j_{1}(0)=-3 / 2\right\}$. Each section of $X_{0}^{1}$ by a level set $\left\{J_{0}=j\right\}$ is a pointed disk containing bounded, hence periodic, trajectories of of the uncontrolled system. The energy is negative but, as $J_{0}=E-C, X_{0}^{1}$ contains both direct $(C>0)$, retrograde $(C<0)$ and collision orbits $(C=0)$. In contrast, the controllability result in [15] was obtained on the elliptic domain, $X_{0} \cap\{E<0, C>0\}$, using the periodicity of the drift and excluding collisions (a sign had then to be imposed on the momentum so that the manifold be connex).

Remark 2. In order to obtain an existence result for minimum time, one has to prove that minimizing trajectories remain into a fixed compact (which depends on the prescribed boundary conditions). Then, from any minimizing sequence one can extract a converging subsequence whose limit is an admissible trajectory by virtue of the convexity of the velocity field $\left\{F_{0}(x)+\varepsilon u_{1} F_{1}(x)+u_{2} F_{2}(x),|u| \leq\right.$ $1\}$ for all $x \in X$ (Filippov theorem). One of the difficulties due to collisions is so to give bounds on the distance to the singularities, $-\mu$ and $1-\mu$. 


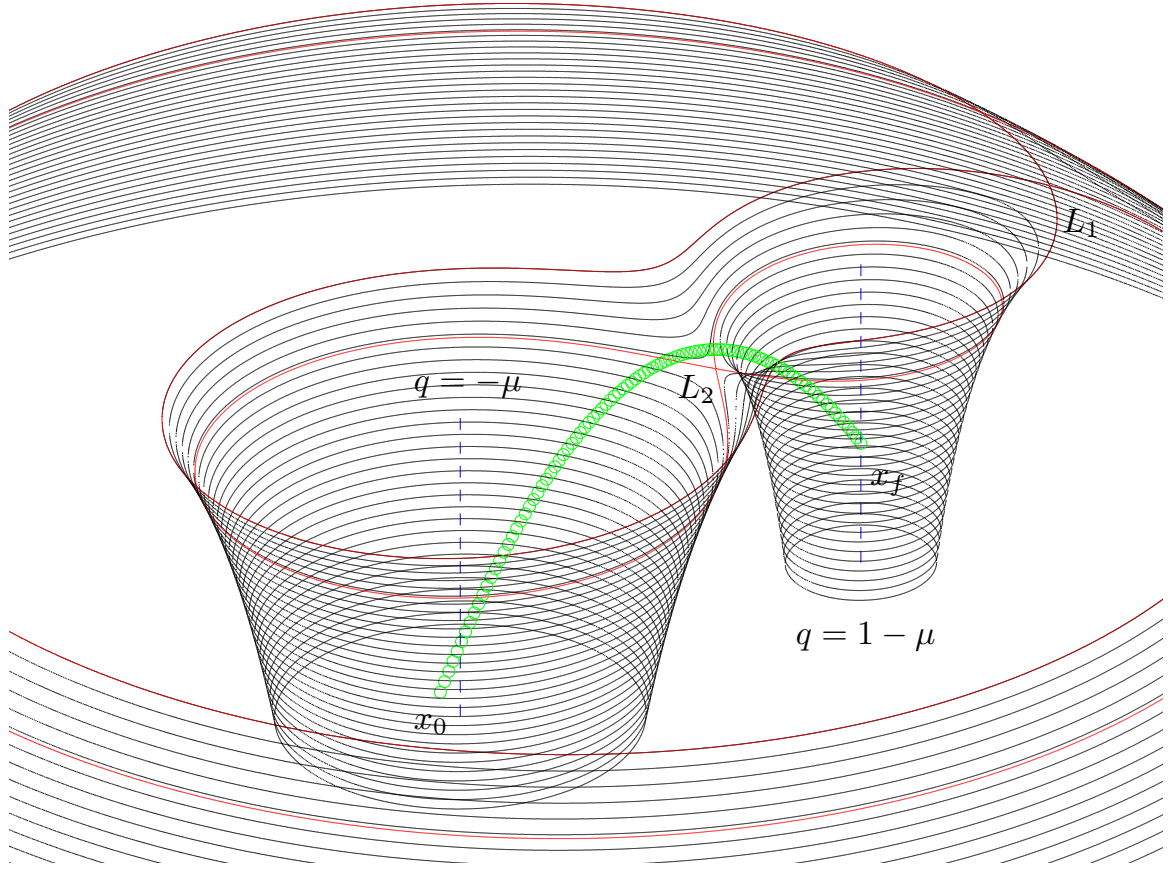

Figure 2: Projection of the open submanifold $X_{\mu}^{1}$ in the $\left(q_{1}, q_{2}, J_{\mu}\right)$-space. The boundary of the volume is an apparent contour generated by the projection. It is the zero velocity set. Above each interior point there is an $\mathbf{S}^{1}$-fibre corresponding to the argument of $\dot{q}$. For $\mu \in(0,1), j_{2}<j_{1}$ and $X_{\mu}^{1}$ is connex. It is necessary that $J_{\mu}$ becomes greater than $j_{2}$ to make the transfer from $x_{0}$ to $x_{f}$. This strategy is observed on time minimum trajectories which pass in the neighbourhood of the $L_{2}$ point (see Fig. 5, Section 4 ).

\section{Singularities of the extremal flow}

Let $u:\left[0, t_{f}\right] \rightarrow \mathbf{R}^{2}$ be a measurable time-minimizing control of the controlaffine system

$$
\dot{x}(t)=F_{0}(x(t))+u_{1} F_{1}(x(t))+u_{2} F_{2}(x(t)), \quad u_{1}^{2}(t)+u_{2}^{2}(t) \leq 1,
$$

defined on a manifold $X$ of dimension four. Let $x$ denote the associated Lipschitz trajectory. Pontrjagin maximum principle [1] asserts that $x$ is the projection of a Lipschitzian function, $z=(x, p):\left[0, t_{f}\right] \rightarrow T^{*} X \backslash 0$, valued in the cotangent bundle minus the null section. The triple $(x, u, p)$ is the reference extremal. In coordinates, there is a nonpositive scalar $p^{0}$ such that, a.e. on $\left[0, t_{f}\right]$,

$$
\dot{x}(t)=\frac{\partial H}{\partial p}(x(t), u(t), p(t)), \quad \dot{p}(t)=-\frac{\partial H}{\partial x}(x(t), u(t), p(t)),
$$

with Hamiltonian $H(x, u, p):=p^{0}+H_{0}(x, p)+u_{1} H_{1}(x, p)+u_{2} H_{2}(x, p)$ and Hamiltonian lifts $H_{i}(x, p):=\left\langle p, F_{i}(x)\right\rangle, i=0,1,2$. Moreover, the maximization condition holds a.e.

$$
H(x(t), u(t), p(t))=\max _{|v| \leq 1} H(x(t), v, p(t)) .
$$


As a result, $H$ is a.e. equal to a constant (zero, here, because the final time is free) along $(x, u, p)$, and

$$
u(t)=\frac{\psi(t)}{|\psi(t)|}
$$

whenever the switching function $\psi(t):=\left(H_{1}, H_{2}\right)(x(t), p(t))$ does not vanish. The switching surface is

$$
\Sigma:=\left\{(x, p) \in T^{*} X \mid H_{1}(x, p)=H_{2}(x, p)=0\right\},
$$

and the crux for regularity is to study contacts (switching points) with $\Sigma$ since, outside the surface, extremals are smooth. Extremals along which $\psi$ does not vanish are bang extremals (denoted $\gamma_{b}$ ), while those on which $\psi$ is identically zero are singular ones (denoted $\left.\gamma_{s}\right)$. We use the notation $F_{i j}:=\left[F_{i}, F_{j}\right]$ (resp. $H_{i j}:=$ $\left.\left\{H_{i}, H_{j}\right\}\right)$ for Lie (resp. Poisson ${ }^{3}$ ) brackets of vector fields (resp. Hamiltonian lifts of these). The following analysis refines the one in [8, 15] using the tools of [9, 22 .

We assume that

(i) $D(x):=\operatorname{det}\left(F_{1}(x), F_{2}(x), F_{01}(x), F_{02}(x)\right) \neq 0, x \in X$.

This assumption, which in particular implies that the span of $F_{1}$ and $F_{2}$ is of constant rank two, so that $\Sigma$ is an embedded codimension two submanifold of the cotangent bundle, is geometric in the following sense. Let $\mathscr{D}$ be a rank 2 distribution over the 4-dimensional manifold $X$ (subbundle of $T X$ with fibres of constant dimension two). Equipped with a Riemannian tensor $g,(\mathscr{D}, g)$ defines a sub-Riemannian structure [3]. Given a vector field $F_{0}$ over $X$, consider the problem of finding the minimum time Lipschitz trajectories subject to

$$
\dot{x}(t)=F_{0}(x(t))+v, \quad v \in \mathscr{D}, \quad|v|_{g}:=\sqrt{g_{x(t)}(v)} \leq 1,
$$

that connect prescribed points of $X$. Given any local frame $\left\{F_{1}, F_{2}\right\}$ of $\mathscr{D}$, orthonormal with respect to $g$, this problem is reformulated as (2). Obviously, the previous assumption only depends on the distribution and on the drift (see also Lemma 3.

Lemma 2. If a singular extremal passes through $z_{0} \in \Sigma$, then $H_{12}\left(z_{0}\right) \neq 0$.

Proof. The switching function is Lipschitz and, almost everywhere,

$$
\begin{aligned}
& \dot{\psi}_{1}(t)=H_{01}(z(t))-u_{2}(t) H_{12}(z(t)), \\
& \dot{\psi}_{2}(t)=H_{02}(z(t))+u_{1}(t) H_{12}(z(t)) .
\end{aligned}
$$

Assume by contradiction $H_{12}\left(z_{0}\right)=0$. Along a singular extremal, $\psi$ vanishes identically and so does $\dot{\psi}$. Then $H_{1}, H_{2}, H_{01}$ and $H_{02}$ vanish at $z_{0}=\left(x_{0}, p_{0}\right)$, which implies $p_{0}=0$ because of assumption (i). This is impossible along a minimum time extremal.

\footnotetext{
${ }^{3}$ The Poisson bracket of two smooth functions $f, g$ on $T^{*} X$ is $\{f, g\}:=\sum_{i}^{n} \partial_{x_{i}} g \partial_{p_{i}} f-$ $\partial_{x_{i}} f \partial_{p_{i}} g, n=\operatorname{dim} X$. In particular, the Poisson bracket of lifts vector fields $F_{i}, F_{j}$ is the lift of their Lie bracket, $\left\{H_{i}, H_{j}\right\}=H_{i j}$.
} 
In the neighbourhood of $z_{0} \in \Sigma$ such that $H_{12}\left(z_{0}\right) \neq 0$, the following dynamical feedback is well defined,

$$
u_{s}(z):=\frac{1}{H_{12}(z)}\left(-H_{02}, H_{01}\right)(z)
$$

(Compare [16] where, on the contrary, singular extremals are studied in the involutive case.) Plugging this control into $H$ sets up a new Hamiltonian,

$$
H_{s}(z):=H\left(z, u_{s}(z)\right)=p^{0}+H_{0}(z)+u_{s, 1}(z) H_{1}(z)+u_{s, 2}(z) H_{2}(z) .
$$

Proposition 1. Let $z_{0} \in \Sigma, H_{12}\left(z_{0}\right) \neq 0$; there is exactly one singular extremal passing through $z_{0}$, and it is defined by the flow of $H_{s}$.

Proof. First we show that $\Sigma$ is invariant with respect to the flow of $H_{s}$. Let $z_{0} \in \Sigma, H_{12}\left(z_{0}\right) \neq 0$, and let $z_{s}$ be the associated integral curve of $H_{s}$ through it. Let $\varphi:=\left(H_{1}, H_{2}\right) \circ z_{s}$; then $\varphi$ is smooth and

$$
\begin{aligned}
\dot{\varphi}_{1}(t) & =\left\{H_{s}, H_{1}\right\}\left(z_{s}(t)\right), \\
& =\underbrace{H_{01}-\left.u_{s, 2} H_{12}\right|_{z_{s}(t)}}_{0}+\left\{u_{s, 1}, H_{1}\right\} H_{1}+\left.\left\{u_{s, 2}, H_{1}\right\} H_{2}\right|_{z_{s}(t)},
\end{aligned}
$$

and similarly for $\dot{\varphi}_{2}$, so $\dot{\varphi}(t)=A(t) \varphi(t)$ with

$$
A(t):=\left[\begin{array}{ll}
\left\{u_{s, 1}, H_{1}\right\} & \left\{u_{s, 2}, H_{1}\right\} \\
\left\{u_{s, 1}, H_{2}\right\} & \left\{u_{s, 1}, H_{2}\right\}
\end{array}\right]\left(z_{s}(t)\right) .
$$

Since $\varphi(0)=\left(H_{1}, H_{2}\right)\left(z_{0}\right)=(0,0), \varphi$ is identically zero and $z_{s}$ remains on $\Sigma$. Now,

$$
H_{s}^{\prime}(z)=\frac{\partial H}{\partial z}\left(z, u_{s}(z)\right)+\frac{\partial H}{\partial u}\left(z, u_{s}(z)\right) u_{s}^{\prime}(z), \quad \frac{\partial H}{\partial u}(z, u)=\left(H_{1}, H_{2}\right)(z),
$$

so $\vec{H}_{s}\left(z_{s}(t)\right)=\vec{H}\left(z_{s}(t), u_{s}\left(z_{s}(t)\right)\right)$ as $\partial H / \partial u$ vanishes along $z_{s}$, and $\left(z_{s}, u_{s} \circ z_{s}\right)$ is extremal.

Consider the stratification of $\Sigma=\Sigma_{-} \cup \Sigma_{0} \cup \Sigma_{+}$where

$$
\begin{aligned}
\Sigma_{-} & :=\left\{z \in \Sigma \mid H_{12}^{2}(z)<H_{01}^{2}(z)+H_{02}^{2}(z)\right\} \\
\Sigma_{0} & :=\left\{z \in \Sigma \mid H_{12}^{2}(z)=H_{01}^{2}(z)+H_{02}^{2}(z)\right\} \\
\Sigma_{+} & :=\left\{z \in \Sigma \mid H_{12}^{2}(z)>H_{01}^{2}(z)+H_{02}^{2}(z)\right\} .
\end{aligned}
$$

We use a nilpotentatization to study the behaviour of bang extremals in the neighbourhood of points in $\Sigma_{-} \cup \Sigma_{+}$.

In the nilpotent approximation around a point $z_{0}=\left(x_{0}, p_{0}\right) \in \Sigma \backslash 0$, Poisson brackets of length greater or equal to three vanish; since the time derivatives of the length two brackets only involve such brackets, $H_{01}, H_{02}$ and $H_{12}$ are constant in this approximation. Under assumption (i), $\left\{F_{1}, F_{2}, F_{01}, F_{02}\right\}$ form a frame so $\left(H_{01}, H_{02}\right)\left(z_{0}\right) \neq(0,0)$ since $p_{0}$ would otherwise be zero. Set

$$
H_{01}\left(z_{0}\right)=: a_{1}, \quad H_{02}\left(z_{0}\right)=: a_{2}, \quad H_{12}\left(z_{0}\right)=: b,
$$




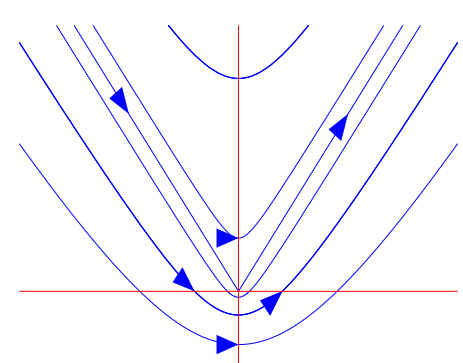

$z_{0} \in \Sigma_{-}$

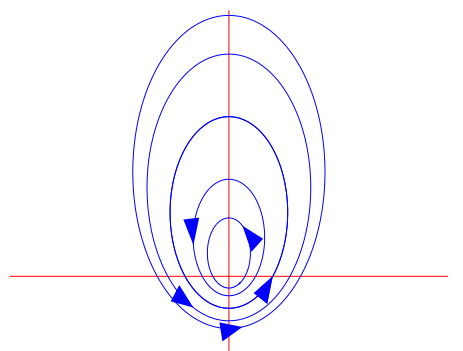

$z_{0} \in \Sigma_{+}$

Figure 3: Phase portraits of the switching function under assumption (i). For $z_{0} \in \Sigma_{-}$, the half-line $\theta=\pi-\theta_{b}\left(z_{0}\right)$ (resp. $\left.\theta=\theta_{b}\left(z_{0}\right)\right)$ goes to the origin (resp. departs from the origin).

with $\left(a_{1}, a_{2}\right) \neq(0,0)$. Making a polar blowing up $\left(\psi_{1}, \psi_{2}\right)=(\rho \cos \theta, \rho \sin \theta)$, the differential equation for the switching function in the nilpotent approximation in the neighbourhood of $z_{0}$ reads

$$
\dot{\rho}(t)=a_{1} \cos \theta(t)+a_{2} \sin \theta(t), \quad \rho(t) \dot{\theta}(t)=b-a_{1} \sin \theta(t)+a_{2} \cos \theta(t),
$$

which, up to some rotation and rescaling, can be normalized to

$$
\dot{\rho}(t)=\cos \theta(t), \quad \rho(t) \dot{\theta}(t)=c-\sin \theta(t),
$$

with $c:=b / \sqrt{a_{1}^{2}+a_{2}^{2}}$. This system is integrated according to

$$
\rho(\theta)=\rho(\theta(0))\left|\frac{c-\sin \theta(0)}{c-\sin \theta}\right|
$$

whence the phase portraits Fig. 3 for $|c|<1\left(z_{0} \in \Sigma_{-}\right)$and $|c|>1\left(z_{0} \in \Sigma_{+}\right)$. When $|c|<1$, the origin is reached along $\theta=\pi-\theta_{b}\left(z_{0}\right)(\dot{\rho}<0)$ and departs from it along $\theta=\theta_{b}\left(z_{0}\right)(\dot{\rho}>0)$ where

$$
\theta_{b}\left(z_{0}\right):=\arcsin \frac{H_{12}}{\sqrt{H_{01}^{2}+H_{02}^{2}}}\left(z_{0}\right) .
$$

Summarizing,

Theorem 2. Let $z_{0} \in \Sigma_{-}$; every extremal is locally of the form $\gamma_{b} \gamma_{s} \gamma_{b}$ ( $\gamma_{s}$ empty if $\left.H_{12}\left(z_{0}\right)=0\right)$; every admissible extremal is locally the concatenation of at most two bang arcs. Let $z_{0} \in \Sigma_{+}$; every extremal is locally bang or singular; every optimal extremal is locally bang. Optimal singular extremals are given by the flow of $H_{s}$ and contained in $\Sigma_{0}$ (saturating).

Proof. A singular extremal passing through $z_{0} \in \Sigma_{-}$cannot be admissible since, if $H_{12}\left(z_{0}\right) \neq 0$, the singular control is well defined but $\left|u_{s}\left(z_{0}\right)\right|>1$. A singular extremal passing through $z_{0} \in \Sigma_{+}$is admissible since $\left|u_{s}\left(z_{0}\right)\right|<1$ but cannot be optimal: As it is interior to the constraint, it must satisfy the Goh second order necessary condition [1],

$$
\left\{\frac{\partial H}{\partial u_{1}}, \frac{\partial H}{\partial u_{2}}\right\}\left(z_{0}, u_{s}\left(z_{0}\right)\right)=H_{12}\left(z_{0}\right)=0,
$$


which is excluded. In the neighbourhood of $\Sigma_{+}$points, the connection between bang and singular extremals is not possible according to the phase portrait in the nilpotent approximation.

An example of saturating singular control is provided by the following nilpotent system (compare [8]). Let

$$
F_{0}(x)=\left(1+x_{3}\right) \frac{\partial}{\partial x_{1}}+x_{4} \frac{\partial}{\partial x_{2}}, \quad F_{1}(x)=x_{4} \frac{\partial}{\partial x_{1}}+\frac{\partial}{\partial x_{3}}, \quad F_{2}(x)=\frac{\partial}{\partial x_{4}} .
$$

One checks that $H_{12}=H_{01}=-p_{1}, H_{02}=-p_{2}$, so $\Sigma_{0}$ is defined by $x_{4} p_{1}+p_{3}=0$, $p_{4}=0$ and $p_{2}=0$. The singular control is $u_{s}(z)=\left(-p_{2} / p_{1}, 1\right)$ and

$$
H_{s}(x, p)=\left(1+x_{3}\right) p_{1}-\frac{p_{2} p_{3}}{p_{1}}+p_{4}
$$

Through $z_{0} \in \Sigma_{0}$ such that $x_{40}=0$ passes the singular extremal

$$
\begin{gathered}
x_{1}(t)=\left(1+x_{30}\right) t+x_{10}, \quad x_{2}(t)=\frac{t^{2}}{2}+x_{20}, \quad x_{3}(t)=x_{30}, \quad x_{4}(t)=t, \\
p_{1}(t)=p_{10} \neq 0, \quad p_{2}(t)=0, \quad p_{3}(t)=-p_{10} t, \quad p_{4}(t)=0,
\end{gathered}
$$

associated with $u_{s}=(0,1)$.

Let us now define

$$
\begin{aligned}
& D_{1}(x):=\operatorname{det}\left(F_{1}(x), F_{2}(x), F_{12}(x), F_{02}(x)\right), \\
& D_{2}(x):=\operatorname{det}\left(F_{1}(x), F_{2}(x), F_{01}(x), F_{12}(x)\right),
\end{aligned}
$$

to strengthen and replace assumption (i) by

(i') $D_{1}^{2}(x)+D_{2}^{2}(x)<D^{2}(x), x \in X$.

This assumption only depends on the sub-Riemannian structure and the drift.

Lemma 3. Assumption ( $\left.i^{\prime}\right)$ is independent of a particular choice of orthonormal frame on $(\mathscr{D}, g)$.

Proof. Let $\left\{F_{1}, F_{2}\right\}$ and $\left\{\hat{F}_{1}, \hat{F}_{2}\right\}$ be two orthonormal bases in the neighbourhood of some point on $x$. There exists a smooth function $\theta$ such that, in coordinates,

$$
\begin{aligned}
& \hat{F}_{1}(x)=\cos \theta(x) F_{1}(x)+\sin \theta(x) F_{2}(x) \\
& \hat{F}_{2}(x)=\varepsilon\left(-\sin \theta(x) F_{1}(x)+\cos \theta(x) F_{2}(x)\right)
\end{aligned}
$$

with $\varepsilon= \pm 1$. One can restrict to $\varepsilon=1$ and use the following facts

$$
[F, \beta G]=[F, G] \quad \bmod \mathbf{R} G, \quad[\alpha F, \beta G]=[F, G] \quad \bmod \operatorname{Span}\{F, G\},
$$

to check that $\hat{D}_{1}=D_{1}, \hat{D}_{2}=D_{2}$ and $\hat{D}=D$ where

$$
\hat{D}_{1}(x):=\operatorname{det}\left(\hat{F}_{1}(x), \hat{F}_{2}(x),\left[\hat{F}_{1}, \hat{F}_{2}\right](x),\left[\hat{F}_{0}, \hat{F}_{2}\right](x)\right), \quad \text { etc. }
$$


Remark 3. When the distribution is involutive (which we shall assume later), $D_{1}=D_{2}=0$ and, with obvious notation, (i') simply asserts that $\mathscr{D}+\left[F_{0}, \mathscr{D}\right]$ has full rank - this is assumption (i). Assumption (i') can so be interpretated as "bounding the non-holonomy" of $\mathscr{D}$ with respect to $F_{0}$.

Corollary 1. Every extremal is locally of the form $\gamma_{b} \gamma_{s} \gamma_{b}$ ( $\gamma_{s}$ possibly empty); for such a sequence, the total angle switch of the control between the entry point into $\Sigma, z_{0}$, and the exit point $z_{0}^{\prime}$ is $\theta_{b}\left(z_{0}\right)+\theta_{b}\left(z_{0}^{\prime}\right)+\pi$. Admissible extremals are the concatenation of finitely many bang arcs.

Proof. Computing,

$$
\begin{gathered}
\operatorname{det}\left(F_{1}(x), F_{2}(x), F_{01}(x)-u_{2} F_{12}(x), F_{02}(x)+u_{1} F_{12}(x)\right)= \\
D(x)-u_{2} D_{1}(x)+u_{1} D_{2}(x), \quad x \in X, \quad u \in \mathbf{R}^{2} .
\end{gathered}
$$

Hence, for $|u| \leq 1,\left\{F_{1}, F_{2}, F_{01}-u_{2} F_{12}, F_{02}+u_{1} F_{12}\right\}$ form a frame by virtue of (i'). Let $z_{0}=\left(x_{0}, p_{0}\right)$ belong to $\Sigma \backslash 0$. For an arbitrary $u \in \mathbf{R}^{2},|u| \leq 1$,

$$
\left[\begin{array}{c}
\left\langle p_{0}, F_{01}\left(x_{0}\right)\right\rangle \\
\left\langle p_{0}, F_{02}\left(x_{0}\right)\right\rangle
\end{array}\right]+\left\langle p_{0}, F_{12}\left(x_{0}\right)\right\rangle\left[\begin{array}{r}
-u_{2} \\
u_{1}
\end{array}\right] \neq\left[\begin{array}{l}
0 \\
0
\end{array}\right],
$$

since $p_{0}$ would otherwise be zero. As a result

$$
H_{12}^{2}\left(z_{0}\right)<H_{01}^{2}\left(z_{0}\right)+H_{02}^{2}\left(z_{0}\right),
$$

so $\Sigma \backslash 0=\Sigma_{-}$and the local structure follows from the previous theorem. If the singular arc is not empty, by virtue of (5) the angle switch at the bang-singular junction is $\left(\varepsilon \pi / 2+\theta_{0}\right)-\left(\pi-\theta_{b}\left(z_{0}\right)+\theta_{0}\right)$ with $\varepsilon:=\operatorname{sign} H_{12}\left(z_{0}\right), \theta_{0}$ being the argument of $\left(H_{01}, H_{02}\right)\left(z_{0}\right) \neq(0,0)$. Similarly, the angle switch at the singularbang junction is $\left(\theta_{b}\left(z_{0}^{\prime}\right)+\theta_{0}^{\prime}\right)-\left(\varepsilon^{\prime} \pi / 2+\theta_{0}^{\prime}\right)$. As $H_{12}$ does not vanish along the singular arc, $\varepsilon=\varepsilon^{\prime}$, whence the result. If the singular arc is empty (notably when $\left.H_{12}\left(z_{0}\right)=0\right)$, the angle switch is $\left(\theta_{b}\left(z_{0}\right)+\theta_{0}\right)-\left(\pi-\theta_{b}\left(z_{0}\right)+\theta_{0}\right)$ and the formula still holds with $z_{0}=z_{0}^{\prime}$.

Assuming moreover that

(ii) $\mathscr{D}$ is involutive,

we get

Corollary 2 (15]). The switching function is continuously differentiable, and every extremal is locally bang-bang with switchings of angle $\pi$ (" $\pi$-singularities").

Proof. For $z_{0} \in \Sigma \backslash 0$, one has $H_{12}\left(z_{0}\right)=0$ because of (ii), so no singular arc passes through a switching point; $\theta_{b}\left(z_{0}\right)=\pi$ and the angle switch is $\pi$. As the bracket $H_{12}$ vanishes at switching points, $\dot{\psi}$ is continuous as observed from (3.4).

In order to give a global bound on the number of switchings, we finally add assumption

(iii) $F_{0} \notin \operatorname{Span}\left\{F_{1}, F_{2}, F_{01}\right\}$, 
and define

$$
\Sigma_{1}:=\Sigma \cap\left\{(x, p) \in T^{*} X \mid F_{0}(x) \in \operatorname{Span}_{x}\left\{F_{1}, F_{2}, F_{02}\right\}\right\} .
$$

Remark 4. (i') + (ii) are equivalent to (i) + (ii). In contrast to (i), (i') and (ii), property (iii) (and $\Sigma_{1}$ ) does depend on the particular choice of orthonormal basis of $\mathscr{D}$.

Theorem 3 (15]). In the normal case, there cannot be consecutive switchings in $\Sigma_{1}$. In particular, if $\Sigma=\Sigma_{1}$, any normal optimal control has at most one switching which is a $\pi$-singularity.

Proof. According to (i), there are continous scalar functions $\lambda_{1}, \lambda_{2}$ on $X$ such that

$$
F_{0}=\lambda_{1} F_{01}+\lambda_{2} F_{02} \bmod \mathscr{D} .
$$

At a switching point $z_{0}=\left(x\left(t_{0}\right), p\left(t_{0}\right)\right) \in \Sigma_{1} \backslash 0$,

$$
H\left(x\left(t_{0}\right), u\left(t_{0}\right), p\left(t_{0}\right)\right)=-p^{0}=\lambda_{2}\left(x\left(t_{0}\right)\right) H_{02}\left(z_{0}\right)=\lambda_{2}\left(x\left(t_{0}\right)\right) \dot{\psi}_{2}\left(t_{0}\right) .
$$

In the normal case, $p^{0}<0$ so

$$
\lambda_{2}\left(x\left(t_{1}\right)\right) \dot{\psi}_{2}\left(t_{1}\right) \lambda_{2}\left(x\left(t_{2}\right)\right) \dot{\psi}_{2}\left(t_{2}\right)=\left(p^{0}\right)^{2}>0
$$

if we assume that $t_{1}<t_{2}$ are consecutive such switching times. Because of (iii), $\lambda_{2}$ never vanishes; by continuity its sign is constant so $\dot{\psi}_{2}\left(t_{1}\right) \dot{\psi}_{2}\left(t_{2}\right)>0$, and the contradiction follows.

Thanks to Lemma 1 , these results apply to the problem under consideration: The circular restricted three-body has bang-bang time-minimizing controls with finitely many $\pi$-singularities. The study of such singularities is important since, in practice, the rotation speed of the thrust is limited. A remarkably simple geometric interpretation of $\Sigma_{1}$ is obtained when restricting to a two-body system, $\mu=0$. This case is not only important in itself as it also corresponds to the initial and final phases of a typical three-body low-thrust transfer. Such a trajectory resembles a heteroclinic trajectory connecting periodic orbits around each one of the primaries (see Fig. 5). We use for the analysis the geometric coordinates and the radial-orthoradial frame introduced in Section 11. We assume the eccentricity positive, $0<e<1$, to obviate the singularity of these coordinates at circular orbits. With $x=(n, e, \theta, l) \in \mathbf{R}_{+}^{*} \times(0,1) \times \mathbf{S}^{1} \times \mathbf{R}$ (and $\tau=l-\theta), p=\left(p_{n}, p_{e}, p_{\theta}, p_{l}\right) \in\left(\mathbf{R}^{4}\right)^{*}$, we set

$$
\alpha:=-\frac{3 n}{1-e^{2}} p_{n}, \quad \beta:=p_{e}, \quad \gamma:=\frac{p_{\theta}}{e}, \quad c:=\cos \tau, \quad s:=\sin \tau,
$$

so $\Sigma$ is defined by the following algebraic system:

$$
\begin{gathered}
\alpha e s+\beta s-\gamma c=0 \\
\alpha(1+e c)+\beta\left(c+\frac{e+c}{1+e c}\right)+\gamma\left(s+\frac{s}{1+e c}\right)=0 \\
c^{2}+s^{2}=1 .
\end{gathered}
$$

Lemma 4. The switching surface $\Sigma$ for $\mu=0$ is stratified as follows: 
(a) If $\gamma=0$, either $(\alpha, \beta)=(0,0)$, or $s=0$ and $\alpha, \beta$ belong to the union of the two distinct lines

$$
(1+e) \alpha+2 \beta=0, \quad(1-e) \alpha-2 \beta=0 .
$$

(b) If $\gamma \neq 0, s$ is not zero and $\alpha, \beta$ are uniquely determined.

Proof. The algebraic system in $\alpha, \beta$

$$
\begin{gathered}
\alpha e s+\beta s=\gamma c \\
\alpha(1+e c)+\beta\left(c+\frac{e+c}{1+e c}\right)=-\gamma\left(s+\frac{s}{1+e c}\right),
\end{gathered}
$$

has determinant

$$
\left|\begin{array}{rr}
e s & s \\
1+e c & c+\frac{e+c}{1+e c}
\end{array}\right|=-\frac{s\left(1-e^{2}\right)}{1+e c},
$$

whence the result.

Proposition 2. In the two-body case $\mu=0$, the subset $\Sigma_{1}$ is the stratum $\{s=0\}$ of $\Sigma$. Accordingly,

$$
\Sigma \cap\left\{p_{\theta}=0\right\}=\Sigma_{1} \cup\left\{\left(p_{n}, p_{e}, p_{\theta}\right)=(0,0,0)\right\} .
$$

Proof. Using the fact that $F_{0} \in \operatorname{Span}\{\partial / \partial l\}$ and that, for smooth functions $f, g$

$$
\left[f F_{0}, g \widetilde{F}_{2}\right]=f g\left[F_{0}, \widetilde{F}_{2}\right] \bmod \operatorname{Span}\left\{F_{0}, \widetilde{F}_{2}\right\}
$$

the condition $\operatorname{det}\left(F_{0}, \widetilde{F}_{1}, \widetilde{F}_{2},\left[F_{0}, \widetilde{F}_{2}\right]\right)=0$ is equivalent to

$$
\left|\begin{array}{rrrr}
0 & e s & 1+e c & -e s \\
0 & s & c+\frac{e+c}{1+e c} & -s-\frac{s\left(1-e^{2}\right)}{(1+e c)^{2}} \\
0 & -c & s+\frac{s}{1+e c} & c+\frac{e+c}{(1+e c)^{2}} \\
1 & 0 & 0 & 0
\end{array}\right|=0
$$

that is to

$$
s=0 \quad \text { or } \quad e(1+e c)=0
$$

so the conclusion follows.

Geometrically, $s=\sin \tau=0$ (that is, $l-\theta=0 \bmod \pi$ ) corresponds to peri and apocenters. The importance of the stratum $\Sigma \cap\left\{p_{\theta}=0\right\}$ comes from the analysis of the system when the control magnitude $\varepsilon$ goes to zero (lowthrust case). When the control is small, not only the time but also the angular length (longitude) needed to connect two Keplerian orbits in the two-body model becomes large. It thus makes sense to use averaging to analyze the behaviour of extremal trajectories. In the case of the minimization of the $\mathrm{L}^{2}$-norm of the control (energy minimization), this approach goes back to 18 (see also 12 for 
a recent treatment of this question). One first uses the fact that, in the planar model, the drift only acts on the longitude,

$$
\dot{l}(t)=\omega(x(t)), \quad \omega(x):=\frac{n W^{2}}{\left(1-e^{2}\right)^{3 / 2}}, \quad W=1+e \cos \tau,
$$

to set $l$ as the new time. In this new parameterization, minimum time extremals are integral curves of the maximized Hamiltonian

$$
\hat{H}(l, \hat{x}, \hat{p}):=\frac{p^{0}}{\omega(l, \hat{x})}+\varepsilon \sqrt{\hat{H}_{1}^{2}(l, \hat{x}, \hat{p})+\hat{H}_{2}^{2}(l, \hat{x}, \hat{p})},
$$

with $\hat{x}=(n, e, \theta), \hat{p}=\left(p_{n}, p_{e}, p_{\theta}\right)$, and

$$
\begin{aligned}
\hat{H}_{1}^{2}(l, \hat{x}, \hat{p})+\hat{H}_{2}^{2}(l, \hat{x}, \hat{p})=\frac{\left(1-e^{2}\right)^{4}}{n^{8 / 3} W^{4}}\left\{\frac{9 n^{2}}{\left(1-e^{2}\right)^{2}}\left(1+2 e \cos \tau+e^{2}\right) p_{n}^{2}\right. \\
-\frac{12 n}{1-e^{2}}(e+\cos \tau) p_{n} p_{e} \\
\left.+\left[1+\frac{2(e+\cos \tau)}{W} \cos \tau+\frac{(e+\cos \tau)^{2}}{W^{2}}\right] p_{e}^{2}\right\}+\cdots
\end{aligned}
$$

where the dots indicate terms in $p_{\theta}^{2}, p_{n} p_{\theta}$ and $p_{e} p_{\theta}$. The averaged Hamiltonian is

$$
\overline{\hat{H}}(\hat{x}, \hat{p}):=\frac{1}{2 \pi} \int_{0}^{2 \pi} \hat{H}(l, \hat{x}, \hat{p}) \mathrm{d} l .
$$

Proposition 3. The argument of pericenter $\theta$ is a cyclic variable of the averaged system. On the stratum $\left\{p_{\theta}=0\right\}$, the integral $\sqrt{6}$ is hyperelliptic of genus 2 .

Proof. As is clear from the whole dynamics in geometric coordinates (see Section 1), only the difference $\tau=l-\theta$ appear in $\hat{H}$. Averaging with respect to $l$-or equivalently to $\tau$-kills terms in $\theta$ and $p_{\theta}$ becomes a linear first integral. On $\left\{p_{\theta}=0\right\}$ then,

$$
\sqrt{\hat{H}_{1}^{2}+\hat{H}_{2}^{2}}=\frac{1}{W^{3}} \sqrt{R(\cos \tau)}
$$

where $R$ is a degree 3 polynomial with coefficients depending nonlinearly on $\hat{x}$ and quadratically on $\hat{p}$. Setting for instance $u=\cos \tau$ leads to

$$
\int \frac{\mathrm{d} u}{1-u^{2}} \sqrt{\left(1-u^{2}\right) R(u)}
$$

which is an integral parameterized by a genus 2 hyperelliptic curve [20].

For circular targets (which are of great practical importance in two or threebody control), the transversality condition is $p_{\theta}=0$. The previous analysis then suggests that, for small control magnitudes, $p_{\theta}$ should also remain small so that the switching structure be close to the one on $\Sigma \cap\left\{p_{\theta}=0\right\}$ : Assuming there are no trivial switchings $\left(p_{n}, p_{e}, p_{\theta}\right)=0$, one would get a global bound on the number of $\pi$-singularities (at most one). Nevertheless, one should notice that, if $p_{\theta}$ is small but not zero, the switchings do not belong to $\Sigma_{1}$ according to Lemma 4 and Proposition 2, Moreover, when studying the convergence of 
the system towards the averaged one, one should take into accout the lack of regularity due to the radicand vanishing. For instance,

$$
\int_{0}^{\pi / 2} \sqrt{\sin ^{2} l+z^{2}} \mathrm{~d} l=|z| E\left(-i z^{-1}\right)
$$

where $E$ is the complete elliptic integral of second kind. Such a function has a $z^{2} \log |z|$ singularity at the origin and is not $\mathscr{C}^{2}$ (logarithmic branch on the second derivative). See [7] for results on averaging of such fast oscillating systems.

\section{Homotopy}

Consider a control problem with smooth data on an $n$-manifold $X$,

$$
\dot{x}(t)=f(x(t), u(t)), \quad u(t) \in U
$$

with cost

$$
\int_{0}^{t_{f}} f^{0}(x(t), u(t)) \mathrm{d} t \rightarrow \min
$$

and prescribed boundary conditions

$$
x(0)=x_{0}, \quad x\left(t_{f}\right)=x_{f} .
$$

For the sake of simplicity $t_{f}$ is supposed to be fixed, but the analyis below can be made with appropriate changes for free final time as well. We also suppose that $U$ is a manifold without boundary. In coordinates, this is assuming that $u$ belongs to some open subset of $\mathbf{R}^{m}$, where $m$ is the dimension of $U$. Regarding the results of the previous section, in the three-body case this amounts to assuming there are no $\pi$-singularities.

Let $\bar{u}:\left[0, t_{f}\right] \rightarrow U$ be a measurable and essentially bounded control, and let $\bar{x}:\left[0, t_{f}\right] \rightarrow X$ be the resulting trajectory. Pontrjagin maximum principle implies that there exists a nonpositive constant $\bar{p}^{0}$ and a Lipschitz covector function $\bar{p}:\left[0, t_{f}\right] \rightarrow\left(\mathbf{R}^{n}\right)^{*}$, not both zero, so that in coordinates on $T^{*} X$,

$$
\dot{\bar{x}}(t)=\frac{\partial H}{\partial p}(\bar{x}(t), \bar{u}(t), \bar{p}(t)), \quad \dot{\bar{p}}(t)=-\frac{\partial H}{\partial x}(\bar{x}(t), \bar{u}(t), \bar{p}(t)),
$$

and

$$
H(\bar{x}(t), \bar{u}(t), \bar{p}(t))=\max _{v \in U} H(\bar{x}(t), v, \bar{p}(t))
$$

a.e. on $\left[0, t_{f}\right]$. Here,

$$
H(x, u, p):=p^{0} f^{0}(x, u)+\langle p, f(x, u)\rangle .
$$

We assume that, on a neighbourhood of the extremal $(\bar{x}, \bar{p})$, the maximized Hamiltonian

$$
(x, p) \mapsto \max _{v \in U} H(x, u, p)
$$

is well defined and smooth; then $(\bar{x}, \bar{p})$ is an integral curve of the maximized function (see [1]), still denoted $H$ (but now depending only on $x$ and $p$ ). We finally make the Legendre regularity assumption that, uniformly on $\left[0, t_{f}\right]$,

$$
\nabla_{u u}^{2} H(\bar{x}(t), \bar{u}(t), \bar{p}(t)) \leq-\alpha I_{m}
$$


for some $\alpha>0$. As a consequence, there must exist in a neighbourhood of the extremal a smooth implicit function $u(x, p)$ solving the first order necessary condition $\nabla_{u} H=0$ such that $\bar{u}(t)=u(\bar{x}(t), \bar{p}(t))$ and $H(x, p)=H(x, u(x, p), p)$. Summarizing, $\bar{p}(0) \in\left(\mathbf{R}^{n}\right)^{*}$ is a zero of the shooting function 4

$$
p_{0} \mapsto x\left(t_{f}, x_{0}, p_{0}\right)-x_{f},
$$

where the exponential mapping

$$
\exp _{x_{0}}:\left(t, p_{0}\right) \mapsto x\left(t, x_{0}, p_{0}\right)
$$

sends a given $p_{0}$ to the $x$-projection of the integral curve at $t$ of the maximized Hamiltonian. Both functions are well defined and smooth on neighbourhoods of $\bar{p}(0)$ and $\left(t_{f}, \bar{p}(0)\right)$, respectively. A time $t_{c}$ is conjugate to 0 along $(\bar{x}, \bar{p})$ whenever $\bar{p}(0)$ is a critical point of $p_{0} \mapsto \exp \left(t_{c}, p_{0}\right)$. The critical value $x_{c}=\exp \left(t_{c}, \bar{p}(0)\right)$ is the corresponding conjugate point. These notions are related to local necessary or sufficient second order conditions of optimality [1, 9. Testing conjugacy is done in practice by a simple rank evaluation (see [8]; see also Fig. 44).

The endpoint mapping

$$
F_{t_{f}, x_{0}}: u \mapsto \hat{x}\left(t_{f}, x_{0}, u\right)
$$

is well defined and smooth on a neighbourhood in $\mathrm{L}^{\infty}\left(\left[0, t_{f}\right], U\right)$ of (the class of $)$ $\bar{u}$ and maps a control to the solution at $t_{f}$ of the augmented system $\left(\hat{x}=\left(x^{0}, x\right)\right)$

$$
\begin{aligned}
\dot{x}^{0}(t) & =f^{0}(x(t), u(t)), \quad t \in\left[0, t_{f}\right] \quad \text { (a.e.) } \\
\dot{x}(t) & =f(x(t), u(t)), \quad x^{0}(0)=0, \quad x(0)=x_{0} .
\end{aligned}
$$

The optimal control $\bar{u}$ must be a critical point $5^{5}$ of the endpoint mapping: $\operatorname{Im} F_{t_{f}, x_{0}}^{\prime}(\bar{u})$ has codimension in $\mathbf{R}^{n}$. If we assume that $\bar{u}$ is a corank one critical point, and moreover that it is analytical (with analytical data for the problem as well), the absence of conjugate time in $\left(0, t_{f}\right)$ is necessary for $\mathrm{L}^{\infty}$-local optimality ${ }^{6}$ Conversely, replacing the corank one and analyticity conditions by the assumption that the extremal is normal $\left(p_{0}<0\right)$, the absence of conjugate time on $\left(0, t_{f}\right]$ is sufficient for $\mathscr{C}^{0}$-local optimality. ${ }^{7}$

Having in mind these connections with second order conditions in optimal control (see also [10]) we consider a one-parameter smooth Hamiltonian,

$$
H: \mathbf{R}^{2 n} \times \mathbf{R} \ni(x, p, \lambda) \mapsto H(x, p, \lambda) \in \mathbf{R} .
$$

Given a positive final time $t_{f}$ and an initial condition $x_{0}$, we define the shootinglike ${ }^{8}$ homotopy function ${ }^{9}$

$$
h\left(p_{0}, \lambda\right):=x\left(t_{f}, x_{0}, p_{0}, \lambda\right)
$$

\footnotetext{
${ }^{4} \mathrm{~A}$ chart $(O, \varphi)$ in the neighbourhood of $x_{f}$ has to be chosen and the definition should read $p_{0} \mapsto \varphi\left(x\left(t_{f}, x_{0}, p_{0}\right)\right)-\varphi\left(x_{f}\right)$. We may actually suppose that $\varphi\left(x_{f}\right)=0$.

${ }^{5}$ This statement, weaker than the maximum principle, is obvious: Were the function a submersion at $\bar{u}$, it would be locally open and would send neighbourhoods of $\bar{u}$ onto neighbourhoods of the augmented state, $\left(\bar{x}^{0}\left(t_{f}\right), \bar{x}\left(t_{f}\right)\right)$. This would contradict $\mathrm{L}^{\infty}$-local optimality.

${ }^{6}$ That is optimality on a neighbourhood of $\bar{u}$ in $\mathrm{L}^{\infty}\left(\left[0, t_{f}\right], U\right)$.

${ }^{7}$ Optimality of the trajectory among all admissible trajectories belonging to some neighbourhood of $\bar{x}$.

${ }^{8}$ The target $x_{f}$ is normalized to 0 .

${ }^{9}$ For the use of homotopy in optimal control (in particular for motion planning), see also
} 
that maps $\left(p_{0}, \lambda\right)$ to the coordinate $x$ of the solution at $t_{f}$ of

$$
\dot{x}(t)=\frac{\partial H}{\partial p}(x(t), p(t), \lambda), \quad \dot{p}(t)=-\frac{\partial H}{\partial x}(x(t), p(t), \lambda)
$$

with initial conditions $x(0)=x_{0}, p(0)=p_{0}$. By restricting it if necessary, we may assume that its domain of definition, $\Omega \subset \mathbf{R}^{n+1}$, is open and made only of regular points of $h$ so that

$$
\operatorname{rank} h^{\prime}\left(p_{0}, \lambda\right)=n, \quad\left(p_{0}, \lambda\right) \in \Omega .
$$

As a consequence, the level set $\{h=0\}$ is a one-dimensional submanifold of $\mathbf{R}^{n+1}$ called the path of zeros. Typically, one knows a zero of $h(., \lambda)$ for, say, $\lambda=0$, and wants to follow this path to reach if possible a zero for a target value of the parameter, $\lambda=1$. For any $c:=\left(p_{0}, \lambda\right) \in \Omega$, $\operatorname{dim} \operatorname{Ker} h^{\prime}(c)=1$ so one can define the (tangent) vector $T(c)$ as being the unique - up to orientationunit vector in the kernel. The orientation is chosen so that the nonvanishing determinant

$$
\operatorname{det}\left[\begin{array}{c}
h^{\prime}(c) \\
{ }^{t} T(c)
\end{array}\right]
$$

has constant sign on each connected component of $\Omega$. This provides a parameterization by arc length of the connected components of $\{h=0\}$ which can be practically computed by integrating the following differential equation 2 (with $'=\mathrm{d} / \mathrm{d} s)$ :

$$
c^{\prime}(s)=T(c(s)), \quad c(0)=c_{0} \in\{h=0\} .
$$

The aim is to classify each component up to diffeomorphisms, knowing that there are only two possibilities [26]: It is diffeomorphic either to $\mathbf{R}$ or to $\mathbf{S}^{1}$.

In such a parameterization, a point $c(\bar{s})=\left(p_{0}(\bar{s}), \lambda(\bar{s})\right)$ in $\{h=0\}$ is a turning point [2] when $\lambda^{\prime}(\bar{s})=0$. This is equivalent to say that

$$
\operatorname{rank} \frac{\partial x}{\partial p_{0}}\left(t_{f}, x_{0}, p_{0}(\bar{s}), \lambda(\bar{s})\right)=n-1,
$$

that is to say that $t_{f}$ is a conjugate time for $\lambda=\lambda(\bar{s})$ (and that $p_{0}(\bar{s})$ and $x\left(t_{f}, x_{0}, p_{0}(\bar{s}), \lambda(\bar{s})\right)$ are the corresponding critical and conjugate point, respectively). A turning point of order one, that is such that $\lambda^{\prime \prime}(\bar{s}) \neq 0$, actually results in a change of variation on $\lambda$, hence the name. We define $\bar{c}=c(\bar{s}) \in\{h=0\}$ to be a first turning point (along the path starting at $c(0))$ if, for all $s \in[0, \bar{s})$, the curve $t \mapsto x\left(t, x_{0}, p_{0}(s), \lambda(s)\right)$ has no conjugate time on $\left(0, t_{f}\right]$.

Theorem 4. Let $c(\bar{s}) \in\{h=0\}$ be a first turning point of order one; then for $s>\bar{s},|s-\bar{s}|$ small enough, there exist conjugate times in $\left(0, t_{f}\right)$.

17. 32 where the point of view is slightly different; the idea is to devise a path lifting equation to construct a path of zeros in the infinite dimensional set of controls. In this setting, the obstructions described at the end of the current section are translated as non degeneracy and non-explosion issues on the so-called Wazewski equation. Assuming more structure than we do on the dynamics (driftless affine control systems are considered), the authors are able to provide conditions in terms of the Lie algebra of controlled vector fields that overcome these difficulties. The emphasis is also on Galerkin procedures to solve the problem. To some extent, the situation is simpler in our case as considering the shooting function instead of the endpoint mapping restricts the problem to finite dimension. 
The next lemmas are necessary to prove this result. We first recall that, at a corank one critical point $\bar{x}$ of a smooth function $g: \mathbf{R}^{n} \rightarrow \mathbf{R}^{n}$, one can define (up to a scalar) the intrinsic second order derivative [5] as

$$
\bar{\mu} g^{\prime \prime}(\bar{x})_{\mid \operatorname{Ker}} g^{\prime}(\bar{x}) \times \operatorname{Ker} g^{\prime}(\bar{x}) \in \mathscr{L}_{2}\left(\operatorname{Ker} g^{\prime}(\bar{x}), \operatorname{Ker} g^{\prime}(\bar{x}) ; \mathbf{R}\right) \simeq \mathbf{R}
$$

where $\bar{\mu} \in\left(\mathbf{R}^{n}\right)^{*}$ is any nonzero covector with kernel $\operatorname{Im} g^{\prime}(\bar{x})$. The critical point is said nondegenerate provided this quantity is not zero.

Lemma 5. The turning point $c(\bar{s})$ is of order one if and only if $p_{0}(\bar{s})$ is a nondegenerate corank one critical point of $p_{0} \mapsto h\left(p_{0}, \lambda(\bar{s})\right)$.

Proof. Differentiating twice $h(c(s))=0$, one gets

$$
h^{\prime \prime}(c(\bar{s})) \cdot\left(c^{\prime}(\bar{s}), c^{\prime}(\bar{s})\right)+h^{\prime}(c(\bar{s})) \cdot c^{\prime \prime}(\bar{s})=0
$$

As $c^{\prime}(\bar{s})=\left(p_{0}^{\prime}(\bar{s}), 0\right), p_{0}^{\prime}(\bar{s})$ generates the kernel of $\partial h / \partial p_{0}\left(p_{0}(\bar{s}), \lambda(\bar{s})\right)$ and

$$
\frac{\partial h}{\partial p_{0}}(c(\bar{s})) \cdot p_{0}^{\prime \prime}(\bar{s})+\frac{\partial h}{\partial \lambda}(c(\bar{s})) \cdot \lambda^{\prime \prime}(\bar{s})=-\frac{\partial^{2} h}{\partial p_{0}^{2}}(c(\bar{s})) \cdot\left(p_{0}^{\prime}(\bar{s}), p_{0}^{\prime}(\bar{s})\right) .
$$

Multiplying both sides by any nonzero $\bar{\mu}$ whose kernel coincides with the image of $\partial h / \partial p_{0}\left(p_{0}(\bar{s}), \lambda(\bar{s})\right)$, one gets

$$
\bar{\mu} \frac{\partial h}{\partial \lambda}(c(\bar{s})) \cdot \lambda^{\prime \prime}(\bar{s})=-\bar{\mu} \frac{\partial^{2} h}{\partial p_{0}^{2}}(c(\bar{s})) \cdot\left(p_{0}^{\prime}(\bar{s}), p_{0}^{\prime}(\bar{s})\right) .
$$

At the turning point $c(\bar{s}) \in \Omega, \partial h / \partial \lambda$ is transverse to the image of $\partial h / \partial p_{0}$ (regularity). So $\bar{\mu} \partial h / \partial \lambda(c(\bar{s})) \neq 0$, and $\lambda^{\prime \prime}(\bar{s})=0$ if and only if the intrinsic second derivative vanishes.

Remark 5. The order one assumption thus puts some restriction on

$$
\frac{\partial^{2} x}{\partial p_{0}^{2}}\left(t_{f}, x_{0}, p_{0}(\bar{s}), \lambda(\bar{s})\right)
$$

It was previously mentioned that the first order derivative with respect to $p_{0}$ of this function is connected with second order optimality conditions. Here we have a condition of order three.

Lemma 6. Let $\bar{x}$ be a corank one critical point of a smooth function $g: \mathbf{R}^{n} \rightarrow$ $\mathbf{R}^{n}$. Then $\bar{x}$ is degenerate if and only if

$$
\left(\operatorname{det} g^{\prime}\right)^{\prime}(\bar{x})=0 \quad \text { on } \quad \operatorname{Ker} g^{\prime}(\bar{x}) \text {. }
$$

Proof. Let $h \in \mathbf{R}^{n}$; one has

$$
\left.\left(\operatorname{det} g^{\prime}\right)^{\prime}(\bar{x}) \cdot h=\operatorname{tr} \widetilde{\left(\widetilde{g^{\prime}(\bar{x})}\right.} \cdot g^{\prime \prime}(\bar{x}) \cdot h\right) .
$$

Since $g^{\prime}(\bar{x})$ is of rank $n-1$, one can find a nonzero vector $\bar{\xi} \in \operatorname{Ker} g^{\prime}(\bar{x})$ (resp. covector $\bar{\mu}$ with kernel $\left.\operatorname{Im} g^{\prime}(\bar{x})\right)$ such that the adjugate matrix

$$
\widetilde{g^{\prime}(\bar{x})}=\bar{\xi} \bar{\mu}
$$


Thus,

$$
\left(\operatorname{det} g^{\prime}\right)^{\prime}(\bar{x}) \cdot h=\sum_{j=1}^{n} \operatorname{tr}\left(\bar{\xi} \bar{\mu} \frac{\partial g^{\prime}}{\partial x_{j}}(\bar{x})\right) h_{j}=\sum_{j=1}^{n} \bar{\mu} \frac{\partial g^{\prime}}{\partial x_{j}}(\bar{x}) \bar{\xi} h_{j}=\bar{\mu} g^{\prime \prime}(\bar{x})(\bar{\xi}, h) .
$$

In particular, for $h=\bar{\xi} \in \operatorname{Ker} g^{\prime}(\bar{x})$,

$$
\left(\operatorname{det} g^{\prime}\right)^{\prime}(\bar{x}) \cdot \bar{\xi}=\bar{\mu} g^{\prime \prime}(\bar{x})(\bar{\xi}, \bar{\xi}),
$$

whence the conclusion.

Remark 6. Under the assumptions of the lemma, $\chi(x, \mu):=\operatorname{det}\left(\mu I_{n}-g^{\prime}(x)\right)$ has root $\mu=0$ for $x=\bar{x}$, with algebraic multiplicity $k \leq n$ (while the geometric multiplicity of 0 , as an eigenvalue of $g^{\prime}(\bar{x})$, is one). By Malgrange preparation theorem [24], there are smooth scalar functions $a_{0}, \ldots, a_{k-1}$ and $b$ such that, in the neighbourhood of $(\bar{x}, 0)$,

$$
\chi(x, \mu)=b(x, \mu)\left(\mu^{k}+a_{k-1}(x) \mu^{k-1}+\cdots+a_{0}(x)\right),
$$

and $a_{0}(\bar{x})=\cdots=a_{k-1}(\bar{x})=0, b(\bar{x}, 0) \neq 0$. Accordingly,

$$
\left(\operatorname{det} g^{\prime}\right)^{\prime}(\bar{x})=b(\bar{x}, 0) a_{0}^{\prime}(\bar{x}) .
$$

The nondegeneracy at $\bar{x}$ is then equivalent to the statement that $\bar{x}$ is not a critical point of $a_{0}$, plus that $g$ and $a_{0}$ are transverse at $\bar{x}$. The quantity $a_{0}$ can be interpretated as a smooth (and signed) singular value of $g^{\prime}$ when $x$ is varied in the neighbourhood of $\bar{x}$, as is clear from the following example. Take $g\left(x_{1}, x_{2}\right)=\left(x_{2}, x_{1}^{2} / 2\right) ; \bar{x}=(0,0)$ is a nondegenerate corank one critical point. In a small enough neighbourhood of $\bar{x}$, the smallest singular value of

$$
g^{\prime}(x)=\left[\begin{array}{rr}
0 & 1 \\
x_{1} & 0
\end{array}\right]
$$

is $\sigma(x)=\left|x_{1}\right|$, which is not differentiable at the critical point. In contrast, $a_{0}(x)=-x_{1}$ is smooth and provides the information needed to check nondegeneracy.

Proof (of Theorem 4). Define the extended homotopy

$$
\widetilde{h}\left(p_{0}, \lambda, t_{c}\right)=\left(x\left(t_{f}, x_{0}, p_{0}, \lambda\right), \operatorname{det} \frac{\partial x}{\partial p_{0}}\left(t_{c}, x_{0}, p_{0}, \lambda\right)\right) .
$$

By assumption, the point $\left(p_{0}(\bar{s}), \lambda(\bar{s}), t_{f}\right)$ belongs to $\{\widetilde{h}=0\}$, and is regular. Indeed, vectors $\left(\delta p_{0}, \delta \lambda, \delta t_{c}\right)$ in the kernel of $\widetilde{h}^{\prime}$ at this point verify $\delta \lambda=0$ and

$$
\begin{gathered}
\frac{\partial h}{\partial p_{0}}\left(p_{0}(\bar{s}), \lambda(\bar{s})\right) \cdot \delta p_{0}=0 \\
\frac{\partial}{\partial p_{0}} \operatorname{det} \frac{\partial x}{\partial p_{0}}\left(t_{f}, x_{0}, p_{0}(\bar{s}), \lambda(\bar{s})\right) \cdot \delta p_{0}+\frac{\partial}{\partial t_{c}} \operatorname{det} \frac{\partial x}{\partial p_{0}}\left(t_{f}, x_{0}, p_{0}(\bar{s}), \lambda(\bar{s})\right) \cdot \delta t_{c}=0 .
\end{gathered}
$$

One has two cases depending on whether the partial derivative with respect to $t_{c}$ vanishes or not in the second equation. If it does not, the kernel is onedimensional and parameterized by

$$
\delta p_{0} \in \operatorname{Ker} \frac{\partial h}{\partial p_{0}}\left(p_{0}(\bar{s}), \lambda(\bar{s})\right) .
$$


If it does, $\delta p_{0}$ has to be zero since otherwise the previous lemmas would imply that

$$
\frac{\partial}{\partial p_{0}} \operatorname{det} \frac{\partial x}{\partial p_{0}}\left(t_{f}, x_{0}, p_{0}(\bar{s}), \lambda(\bar{s})\right) \cdot \delta p_{0} \neq 0
$$

because of the order one assumption on the turning point. So the kernel of $\widetilde{h}^{\prime}$ is parameterized by $\delta t_{c} \in \mathbf{R}$ and is also of dimension one. The extended homotopy is therefore well defined and regular in a neighbourhood of $\left(p_{0}(\bar{s}), \lambda(\bar{s}), t_{f}\right)$. Parameterizing by arc length, $\sigma$, on $\{\widetilde{h}=0\}$, one has $t_{c}(\bar{\sigma})=t_{f}$. Since the point is a first turning point, it is enough to prove that $t_{c}^{\prime}(\bar{\sigma}) \neq 0$ (here $\left.{ }^{\prime}=d / d \sigma\right)$; then necessarily $t_{c}^{\prime}(\bar{\sigma})<0$, as there would otherwise be conjugate times on $\left(0, t_{f}\right)$ for $\sigma<\bar{\sigma}$ in $\{\widetilde{h}=0\}$, that is for $s<\bar{s}$ in $\{h=0\}$. According to the description of the kernel,

$$
\frac{\partial}{\partial p_{0}} \operatorname{det} \frac{\partial x}{\partial p_{0}}\left(t_{f}, x_{0}, p_{0}(\bar{\sigma}), \lambda(\bar{\sigma})\right) \cdot p_{0}^{\prime}(\bar{\sigma})+\frac{\partial}{\partial t_{c}} \operatorname{det} \frac{\partial x}{\partial p_{0}}\left(t_{f}, x_{0}, p_{0}(\bar{\sigma}), \lambda(\bar{\sigma})\right) \cdot t_{c}^{\prime}(\bar{\sigma})=0 .
$$

The two previous alternatives result either in $p_{0}^{\prime}(\bar{\sigma})$ being non-zero, in which case neither the first term in the sum nor $t_{c}^{\prime}(\bar{\sigma})$ can vanish; or in $p_{0}^{\prime}(\bar{\sigma})=0$, so $\left|t_{c}^{\prime}(\bar{\sigma})\right|=1$ (unit tangent vector). In both situations we conclude that $t_{c}^{\prime}(\bar{\sigma})$ cannot be zero.

In addition to turning points, there are two other issues on homotopy. First, when the connected component of the path considered is diffeomorphic to $\mathbf{R}$, boundary points (if any) are critical points of $h$. The classification of points in $\partial \Omega$ starts with the following result which is a simple consequence of Morse lemma.

Proposition $4([2])$. Let $\bar{c} \in\{h=0\}$ be a nondegenerate hyperbolic corank one critical point of $h$. Then, there are coordinates $d_{1}, \ldots, d_{n+1}$ such that, in the neighbourhood of $\bar{c},\{h=0\}$ is equal to

$$
d_{1}^{2}-d_{2}^{2}=0, \quad d_{3}=\cdots=d_{n+1}=0 .
$$

In this case, we have a critical point jointly in $\left(p_{0}, \lambda\right)$ and the intrinsic second order derivative is, up to a scalar,

$$
\bar{\mu} h^{\prime \prime}(\bar{c})_{\mid \operatorname{Ker} h^{\prime}(\bar{c}) \times \operatorname{Ker} h^{\prime}(\bar{c})} \in \operatorname{Sym}(2, \mathbf{R}) \subset \mathbf{M}(2, \mathbf{R}) \simeq \mathscr{L}_{2}\left(\operatorname{Ker} h^{\prime}(\bar{c}), \operatorname{Ker} h^{\prime}(\bar{c}) ; \mathbf{R}\right)
$$

where $\bar{\mu} \in\left(\mathbf{R}^{n}\right)^{*}$ is any nonzero covector with kernel $\operatorname{Im} h^{\prime}(\bar{c})$. Hyperbolicity means that this order 2 symmetric matrix is nondegenerate with eigenvalues of opposite signs. As a consequence, the path of zeros is locally made of two smooth curves intersecting transversally, resulting in a bifurcation. The last issue is due to global features in parametric optimal control. For a given value $\lambda_{0}$ of the parameter, one has to compare the costs associated to zeros in each connected component of $\{h=0\} \cap\left\{\lambda=\lambda_{0}\right\}$. Each zero of the shooting homotopy function defines an extremal and global solutions, if any, are those giving the infimum of the cost among them. In the three-body problem, the topology of the state manifold, $X_{\mu}=T^{*} Q_{\mu}$, comes into play; $Q_{\mu}$ has the topology of the eight curve with $\pi_{1}\left(Q_{\mu}\right)=\mathbf{Z} * \mathbf{Z}$, and a heuristic classification of extremals based on homology is proposed in 13 .

Two homotopies are used to compute numerically minimum time trajectories of the restricted three-body problem. A continuation on the ratio of masses, 
$\mu$, is first considered. In practice, the isolated contacts with the codimension two switching surface are neglected, and we restrict the computation to smooth extremals without $\pi$-singularities. This yields regularity of the $\mu$-parameterized minimum time problem in view of

Lemma 7. In the absence of $\pi$-singularities, Legendre condition holds.

Proof. If the switching function never vanishes, one has $|u|=1$ everywhere. So we can restrict the control set $U$ to $\mathbf{S}^{1}$. The manifold is without boundary and, in the chart $u=(\cos \alpha, \sin \alpha), \alpha \in \mathbf{R}$, one has ${ }^{10}$

$$
H(x, \alpha, p)=p^{0}+H_{0}(x, p)+\varepsilon\left(\cos \alpha H_{1}(x, p)+\sin \alpha H_{2}(x, p)\right) .
$$

Accordingly,

$$
\nabla_{\alpha \alpha}^{2} H(x, \alpha, p)=-\varepsilon\left(\cos \alpha H_{1}(x, p)+\sin \alpha H_{2}(x, p)\right) .
$$

Along an extremal, $\nabla_{\alpha \alpha}^{2} H(x(t), \alpha(t), p(t))=-\varepsilon|\psi(t)|$ which is bounded over by some negative constant on $\left[0, t_{f}\right]$ as $\psi$ is smooth and nonvanishing.

Using previous knowledge on the two-body minimum time trajectories [14, 15, we are able to compute transfers from a circular orbit around the first primary towards the $L_{2}$ point when $\mu=0$, then to follow the path until any value $\mu \in$ $(0,1)$. The absence of conjugate points - ensuring local optimality - is checked along the path using the hampath code [11] that embeds the relevant rank test, see Fig. 4. A continuation on the target eventually allows to obtain solutions, for instance in the Earth-Moon system $(\mu \simeq 1.21 e-2)$ from a geostationary orbit to a circular lunar one for average values of the control magnitude $\varepsilon$, see Fig. 5. To reach lower values of $\varepsilon$, a continuation on this parameter is finally employed as in 14, which emphasizes the role of the topology of the state space previously mentioned. Many local minima exist, yielding as many zeros of the shooting function. When decreasing $\varepsilon$, at some point on the resulting path the number of revolutions around the first primary has to be increased to retain global optimality, which means using a heuristic to jump to another connected component (branch) of the zero set (see Fig. 6). The situation is analogous to the one in Riemannian geometry with cut and conjugate points: Up to some point, the path provides minimizers; then global optimality is lost (typically because of the topology of the manifold), but local optimality persists up to another point; past this second point (a turning point, in the simple case we framed in the beginning of the section), even local optimality is lost (see Fig. 7). Table 1 summarizes the results obtained.

The computations combining shooting and homotopy presented here are meant to initialize the solution of more complicated problems. A time minimum trajectory of the three-dimensional model of the SMART-1 mission 29 is given Fig. 8 The 3D case proves to be much more difficult to solve numerically than the coplanar one; this is probably due to the angle between the planes containing the initial and final orbit. Multiple shooting provided by hampath and initialized by results on the coplanar model is used; two arcs are considered with a junction point in the neighbourhood the $L_{2}$ Lagrange point. Work in progress includes the treatment of the maximization of the final mass.

\footnotetext{
${ }^{10}$ The Hamiltonian lift $H_{0}$ implicitly depends on $\mu$, since $F_{0}=\overrightarrow{J_{\mu}}$ (compare Section 1 .
} 




Figure 4: Conjugate point computation (rotating frame). Extremals (here projected on the $\left(q_{1}, q_{2}\right)$-space) from a circular orbit around the first primary towards the $L_{2}$ Lagrange point are extended beyond the target. Conjugate points, in red, appear after $t_{f}$, ensuring local optimality. Green dots indicate isocost (isotime) lines.

\section{Conclusion}

In this paper, we have given a controllability result for the restricted threebody problem; under mild assumptions, two orbits around the primaries with Jacobian constants less than the Jacobian at the $L_{1}$ Lagrange point can be connected. Using the control-affine structure of the dynamics, we have given a primary classification of extremals and provided global bounds on the number of switchings of time minimizing controls. Homotopy techniques are instrumental in solving numerically the problem which has natural small parameters; the 
Table 1: Earth-Moon system $(\mu \simeq 1.21 e-2)$. Minimum time $t_{f}$ from the geostationary orbit to the $L_{2}$ Lagrange point, and first conjugate time, $t_{1 c}$. That $t_{1 c}>t_{f}$ ensures local optimality of the computed extremal.

\begin{tabular}{ccc}
$\varepsilon$ & $t_{f}$ & $t_{1 c}$ \\
\hline 2.4405 & 1.4705 & 2.2750 \\
0.2440 & 8.4401 & 10.640 \\
0.2221 & 9.7710 & 12.045 \\
0.2026 & 11.152 & 13.500 \\
0.1806 & 13.157 & 15.595 \\
0.1586 & 14.369 & 16.900 \\
0.1293 & 18.024 & 20.700 \\
0.1074 & 21.323 & 24.125 \\
0.0732 & 32.216 & 35.295 \\
0.0437 & 51.504 & 54.930
\end{tabular}
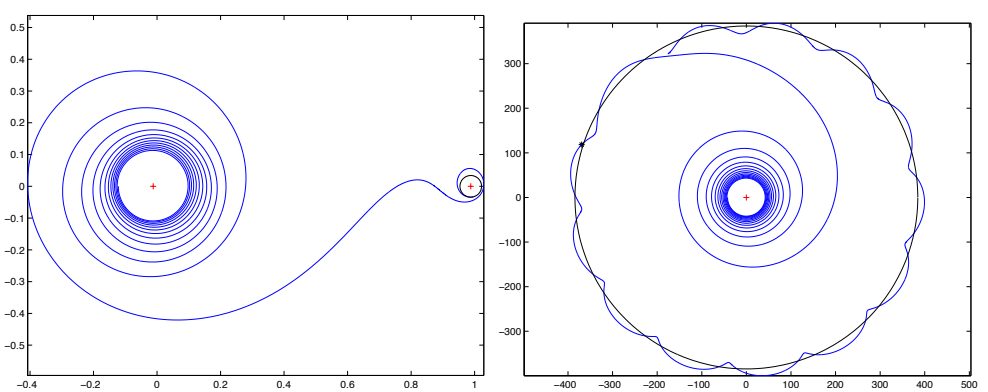

Figure 5: Minimum time trajectory in the Earth-Moon system $(\mu \simeq 1.21 e-2$, $\varepsilon=2.44 e-1)$. Left, in the rotating frame; right, in the fixed frame to emphasize capture by the second primary at the end of the transfer. Before the capture, the trajectory approaches the projection of the $L_{2}$ point in the $\left(q_{1}, q_{2}\right)$-plane.

link between turning points and local optimality of extremals along the path of zeros of a parameterized shooting function has been established in a simple framework. This preliminary analysis of the problem has allowed us to compute minimum time solutions for the boundary conditions of the SMART-1 mission using a two to three-body continuation.

Future work could be devoted to reaching very low thrusts, typical of this kind of mission. The performance index should also be changed to consider instead minimization of the fuel consumption, equivalent to minimizing the $\mathrm{L}^{1}$ norm of the control. The final time should then be fixed, $t_{f}=c \cdot \bar{t}_{f}, c \geq 1$, where $\bar{t}_{f}$ is the minimum time for the prescribed boundary conditions. The additional difficulty in this problem comes from the existence, for $c>1$, of balistic arcs $u=0$ (zero-bang structure of $|u|$ ). A related issue would be to use a 4 -body model so as to include the existence of "cheap" trajectories 25] whose existence rely on the presence of the fourth body. 




Figure 6: Minimum time trajectory for $\varepsilon$ between $2.44 e-1$ and $2.196 e-1$ ( $\mu \simeq 1.21 e-2$, rotating frame). As the control magnitude is decreased, strategies are evolved. In the upper graphs, the first two extremals have the same rotation number around the first primary and both wind around the second one positively. Conversely, the third extremal (bottom left) winds negatively around the second primary, while the fourth (bottom right) makes an additional revolution around the first one.

\section{Acknowledgements}

The authors thank Joseph Gergaud for introducing them to homotopy in optimal control, and Bernard Bonnard for his geometric influence on this work.

\section{References}

[1] Agrachev, A. A.; Sachkov, Y. L. Control Theory from the Geometric Viewpoint, Springer, 2004.

[2] Allgower, E. L.; Georg, K. Introduction to numerical continuation methods, SIAM, 2003.

[3] Bellaïche, A.; Risler, J.-J. Sub-Riemannian geometry, Birkhäuser, 1996.

[4] Betts, J. T.; Erb, S. O. Optimal Low Thrust Trajectories to the Moon. SIAM J. Appl. Dyn. Syst. 2 (2003), no. 2, 144-170.

[5] Boardman, J. M. Singularities of differentiable maps. Publ. Math. IHES, 33 (1967), 383-419.

[6] Bombrun, A.; Chetboun, J.; Pomet, J.-B. Transfert Terre-Lune en poussée faible par contrôle feedback. La mission SMART-1. INRIA Research report (2006), no. 5955, 1-27. 


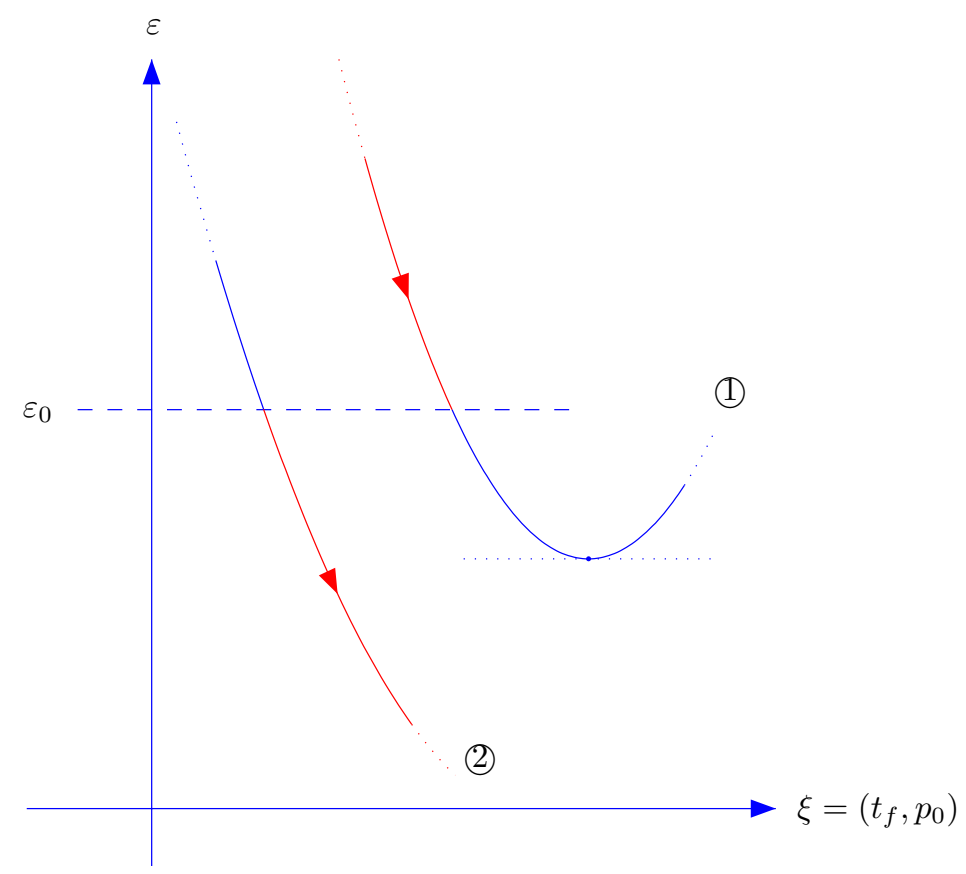

Figure 7: Continuation on $\varepsilon$. Down to $\varepsilon_{0}$, branch 1 yields minimizers. In the case of free final time, the shooting unknown is $\xi=\left(t_{f}, p_{0}\right), p_{0}$ belonging to the zero level set of the Hamiltonian. Past $\varepsilon_{0}$, global optimality is lost on branch 1 and a switch to branch 2 has to be made (resulting in a loss of regularity of the value function). Past the turning point on branch 1 (conjugacy of the target point), even local optimality is lost.

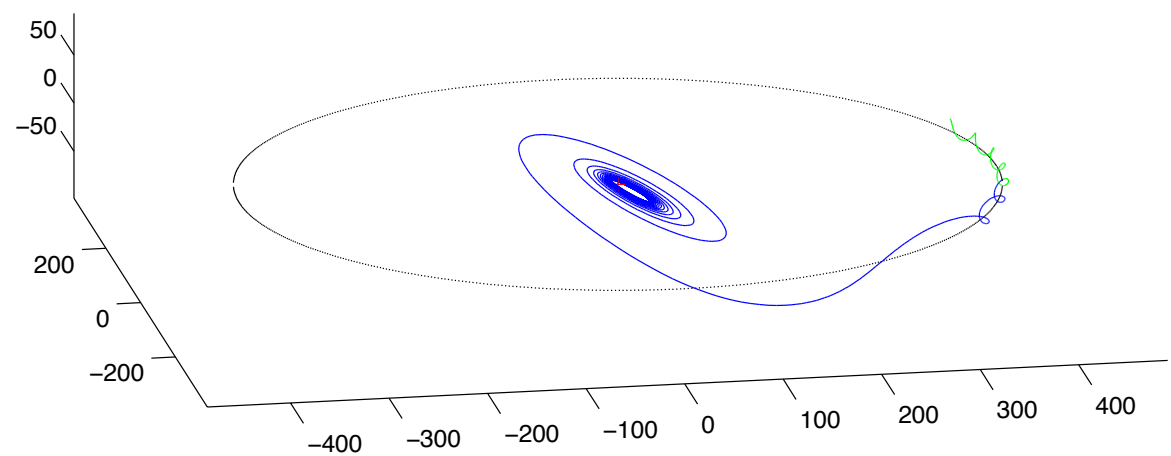

Figure 8: Three-dimensional minimum time transfer, SMART-1 boundary conditions (fixed frame). The control magnitude $\varepsilon$ is 0.7 Newtons for an initial mass of 350 Kilograms and a specific impulse of 1640 seconds (the variation of mass has been taken into account for this simulation, see [29]). The final time is 26.2 days. The dotted black circle represents the orbit of the Moon. The green trajectory represents the uncontrolled motion after capture by the Moon. 
[7] Bombrun, A.; Pomet, J.-B. The averaged control system of fast oscillating control systems. HAL preprint (2011), no. 00648330, 1-27.

[8] Bonnard, B.; Caillau, J.-B.; Trélat, E. Geometric optimal control of elliptic Keplerian orbits. Discrete Contin. Dyn. Syst. Ser. B 5 (2005), no. 4, 929 956.

[9] Bonnard, B.; Chyba, M. Singular trajectories and their role in control theory, Springer, 2003.

[10] Bonnard, B.; Shcherbakova, N.; Sugny, D. The smooth continuation method in optimal control with an application to quantum systems. ESAIM Control Optim. and Calc. Var. 17 (2010), no. 1, 267-292.

[11] Caillau, J.-B.; Cots, O.; Gergaud, J. Differential continuation for regular optimal control problems, Optim. Methods Softw. 27 (2012), no. 2, 177-196. apo.enseeiht.fr/hampath

[12] Caillau, J.-B.; Daoud, B.; Gergaud, J. On some Riemannian aspects of two and three-body controlled problems. Recent Advances in Optimization and its Applications in Engineering, 205-224, Springer, 2010. Proceedings of the 14th Belgium-Franco-German conference on Optimization, Leuven, September 2009.

[13] Caillau, J.-B.; Daoud, B.; Gergaud, J. Discrete and differential homotopy in circular restricted three-body control. Discrete Contin. Dyn. Syst. suppl. (2011), 229-239. Proceedings of 8th AIMS Conference on Dynamical Systems, Differential Equations and Applications, Dresden, May 2010.

[14] Caillau, J.-B.; Gergaud, J.; Noailles, J. 3D Geosynchronous Transfer of a Satellite: Continuation on the Thrust. J. Optim. Theory Appl. 118 (2003), no. $3,541-565$.

[15] Caillau, J.-B.; Noailles, J. Coplanar control of a satellite around the Earth. ESAIM Control Optim. and Calc. Var. 6 (2001), 239-258.

[16] Chittaro, F. C.; Stefani, G. Singular extremals in multi-input time-optimal problem: A sufficient condition. Control Cybernet. 39 (2010), no. 4, 1029 1068.

[17] Chitour, Y. A continuation method for motion-planning problems. \{em ESAIM Control Optim. Calc. Var.\} 12 (2006), no. 1, 139-168.

[18] Edelbaum, T. N. Optimal low-thrust rendez-vous and station keeping. AIAA J. 2 (1964), no. 7, 1196-1201.

[19] Gómez, G.; Jorba, A.; Madesmont, J.; Simó, C. Dynamics and mission design near libration points Vol. III. Advanced methods on collinear points. World Scientific Publishing, 2001.

[20] Jones, G. A.; Singerman, D. Complex Functions. An Algebraic and Geometric Viewpoint. Cambridge University Press, 1987.

[21] Jurdjevic, V. Geometric control theory, Cambridge University Press, 1996. 
[22] Kupka, I. Generalized Hamiltonians and optimal control: A geometric study of extremals. Proceedings of the International Congress of Mathematicians, 1180-1189, Berkeley, 1987.

[23] Kupka, I.; Sallet, G. A sufficient condition for the transitivity of pseudosemigroups: Application to system theory. J. Differential. Eq. 47 (1983), $462-470$.

[24] Malgrange, B. Ideals of differentiable functions. Oxford University Press, 1967.

[25] Marsden, J. E.; Ross, S. D. New methods in celestial mechanics and mission design. Bull. Amer. Math. Soc. (N.S.) 43 (2006), no. 1, 43-73.

[26] Milnor, J. W. Topology from the Differentiable Viewpoint, Princeton University Press, 1997.

[27] Mingotti, G.; Topputo, F.; Bernelli-Zazzera, F. Low-energy, low-thrust transfers to the Moon. Celest. Mech. Dyn. Astr. 105 (2009), 61-74.

[28] Ozimek, M. T.; Howell, K. C. Low-thrust transfers in the Earth-Moon system, including applications to libration point orbits. J. Guidance Control Dynam. 33 (2010), no. 2, 533-549.

[29] Racca, G.; et al. SMART-1 mission description and development status. Planetary and space science 50 (2002), 1323-1337.

[30] Siegel, C. L.; Moser, J. K. Lectures on celestial mechanics. Berlin, 1971.

[31] Sussmann, H. Orbits of families of vector fields and integrability of distributions. Trans. Amer. Math. Soc. 180 (1973), 171-188.

[32] Sussmann, H. A continuation method for nonholonomic path-finding problems. IEEE Publications (1993), 2718-2723. Proceedings of the 32nd IEEE CDC, San Antonio, December 1993.

[33] Szebehely, V. Theory of orbits: The restricted problem of three bodies, Academic Press, 1967. 